\title{
A rational design of a multi-epitope vaccine against SARS-CoV-2 which accounts for the glycan shield of the spike glycoprotein
}

William R. Martin ${ }^{1}$ and Feixiong Cheng ${ }^{1-3}$

${ }^{1}$ Genomic Medicine Institute, Lerner Research Institute, Cleveland Clinic, Cleveland, $\mathrm{OH}$ 44195, USA

${ }^{2}$ Department of Molecular Medicine, Cleveland Clinic Lerner College of Medicine, Case Western Reserve University, Cleveland, OH 44195, USA

${ }^{3}$ Case Comprehensive Cancer Center, Case Western Reserve University School of Medicine, Cleveland, $\mathrm{OH} 44106$, USA

*To whom correspondence should be addressed:

Feixiong Cheng, $\mathrm{PhD}$

Lerner Research Institute, Cleveland Clinic

Tel: +1-216-444-7654; Fax: +1-216-636-0009

Email: chengf@ccf.org 


\section{ABSTRACT}

The ongoing global health crisis caused by Severe Acute Respiratory Syndrome Coronavirus 2 (SARS-CoV-2), the virus which leads to Coronavirus Disease 2019 (COVID-19) has impacted not only the health of people everywhere, but the economy in nations across the world. While vaccine candidates and therapeutics are currently undergoing clinical trials, there is yet to be a proven effective treatment or cure for COVID19. In this study, we have presented a synergistic computational platform, including molecular dynamics simulations and immunoinformatics techniques, to rationally design a multi-epitope vaccine candidate for COVID-19. This platform combines epitopes across Linear B Lymphocytes (LBL), Cytotoxic T Lymphocytes (CTL) and Helper T Lymphocytes (HTL) derived from both mutant and wild-type spike glycoproteins from SARS-CoV-2 with diverse protein conformations. In addition, this vaccine construct also takes the considerable glycan shield of the spike glycoprotein into account, which protects it from immune response. We have identified a vaccine candidate (a $35.9 \mathrm{kDa}$ protein), named COVCCF, which is composed of $5 \mathrm{LBL}, 6 \mathrm{HTL}$, and $6 \mathrm{CTL}$ epitopes from the spike glycoprotein of SARS-CoV-2. Using multi-dose immune simulations, COVCCF induces elevated levels of immunoglobulin activity ( $\lg M, \lg G 1, \lg G 2)$, and induces strong responses from $\mathrm{B}$ lymphocytes, CD4 T-helper lymphocytes, and CD8 T-cytotoxic lymphocytes. COVCCF induces cytokines important to innate immunity, including IFN- $\gamma$, IL4, and IL10. Additionally, COVCCF has ideal pharmacokinetic properties and low immune-related toxicities. In summary, this study provides a powerful, computational vaccine design platform for rapid development of vaccine candidates (including COVCCF) for effective prevention of COVID-19. 


\section{INTRODUCTION}

The current Coronavirus Disease 2019 (COVID-19) pandemic has brought the world to a near standstill, with over 10 million cases worldwide and over 500,000 deaths as of June 29,2020 . While some countries have been able to manage cases using a combination of stay-at-home orders, social distancing, and mask usage, the worldwide 7-day moving average for worldwide cases is currently over 170,000 per day (source: Worldometers.info), indicative of a need for effective prevention and/or treatment of COVID-19. As June 29, 2020, the United States (U.S.) alone has more than 2.6 million confirmed cases, with a death toll of more than 120,000, accompanied by unprecedented social and economic consequences (coronavirus.jhu.edu). However, there are currently no U.S. FDA-approved vaccines for COVID-19, nor are there any proven effective treatments, though clinical trials are underway currently for both.

Key to the interaction between Severe Acute Respiratory Syndrome Coronavirus 2 (SARS-CoV-2), the virus which causes COVID-19, and the human cell is the spike glycoprotein (S protein) ${ }^{1}$, which in SARS-CoV-2 interacts with angiotensin-converting enzyme 2 (ACE2) via its receptor binding domain (RBD) ${ }^{2}$. The $S$ protein is a $180 \mathrm{kDa}$ homotrimer consisting of two subunits, S1 and S2, which mediate attachment to ACE2 and membrane fusion, respectively ${ }^{3}$. The $\mathrm{S} 1$ subunit consists of an $\mathrm{N}$-terminal domain (NTD) and the RBD, while the S2 subunit is composed of a fusion protein (FP), two heptad repeat domains (HR1 and HR2), a transmembrane domain (TM), and a cytoplasmic domain $(\mathrm{CP})^{4}$. In order to fuse its viral membrane with the host cell, the $\mathrm{S}$ protein must be activated at the $\mathrm{S} 1 / \mathrm{S} 2$ boundary ${ }^{5}$. This priming of the $S$ protein is accomplished through the use of the cellular protein TMPRSS2 ${ }^{2}$. 
Because of this, the $S$ protein has been a target for therapeutics ${ }^{4,6}$, including vaccines $^{7}$. However, key to the S protein's ability to ward off an immune response is its considerable glycan shield ${ }^{8,9}$. The glycosylation of the $S$ glycoprotein creates somewhat of a barrier around the spike, preventing immune molecules from reaching the protein surface. Here, we have constructed a multi-epitope vaccine candidate using molecular dynamics simulations and immunoinformatics techniques while considering the impact of the glycan shield on the ability for a particular epitope to elicit an immune response (Figure 1). Selecting only epitopes which would be accessible in an immune response should improve the effectiveness of a vaccine. Our inclusion of multiple conformations for the spike glycoprotein, both in the wild-type as well as in 9 different mutated states, allowed for a broader reach with respect to B-cell epitope prediction; in fact, nearly $75 \%$ of the predicted epitopes were exclusive to the predictions from the mutated systems. Additionally, this method is superior to a sequence-based prediction; a preliminary test for linear B-lymphocyte epitopes over the 1,120 amino acid sequence available via crystal structure using BepiPred $2.0^{10}$ yielded only 27 potential epitopes of length 6 or longer. This, along with careful consideration of the impact glycosylation will have on the ability for a particular epitope to elicit a useful immune response, allowed for the construction of our 331 amino acid vaccine construct, named COVCCF; the physiochemical properties and simulated immunogenicity indicate the potential for the induction of a strong immune response, which would also portend immunity toward the spike glycoprotein of SARSCoV-2. 


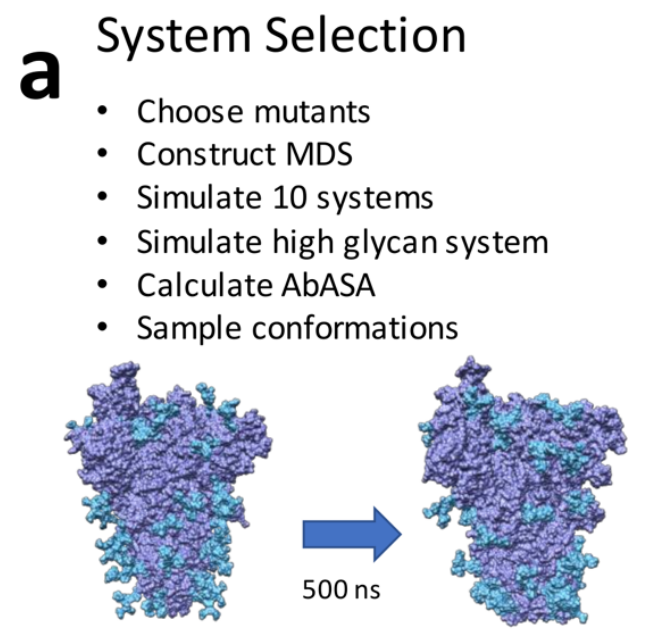

b Epitope Prediction

- Linear B Lymphocytes

- Antigenicity

- Allergenicity

- Toxicity

- Cytotoxic T Lymphocytes

- Immunogenicity

- Antigenicity

- Allergenicity

- Toxicity

- Helper T Lymphocytes

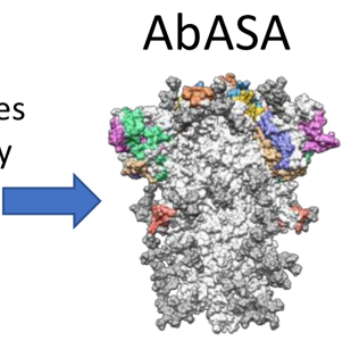

- IFN- $\gamma$

- IL-4

- IL-10

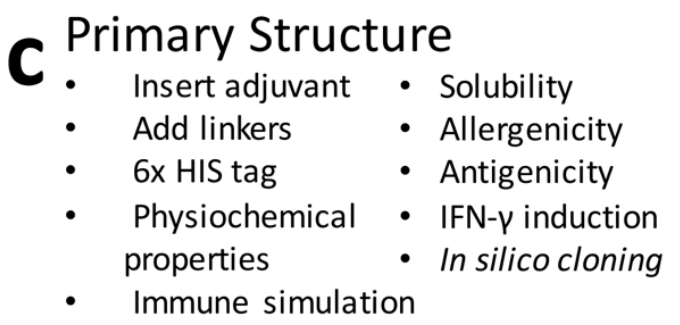

d Secondary/Tertiary Structure

- Predict secondary structure

- Predict tertiary structure

- Tertiary structure refinement

- Protein-protein docking
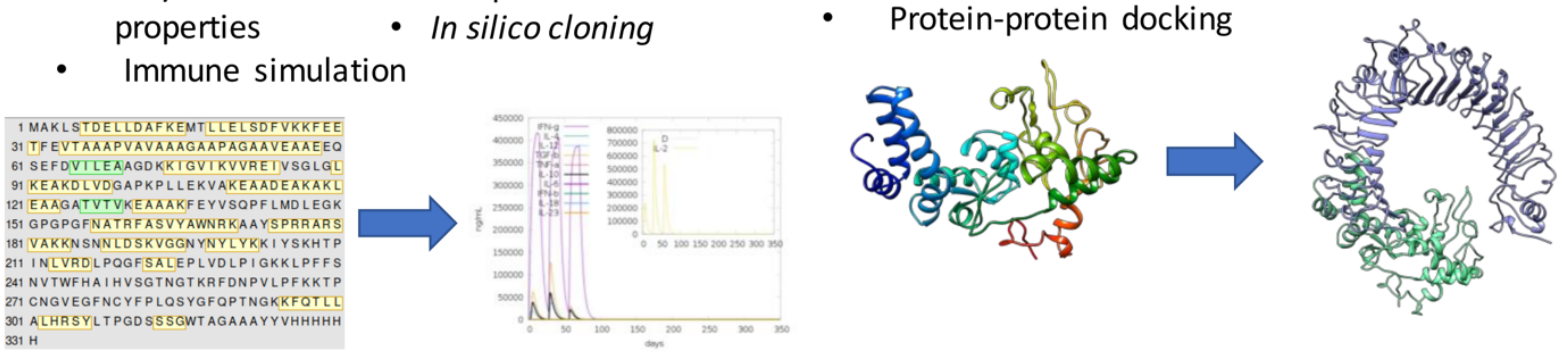

Figure 1: Overall workflow for project. a) Selection of the systems used to generate conformations to be used in the linear B lymphocyte prediction. b) Epitope predictions, including linear B lymphocyte, cytotoxic T lymphocyte, and helper T lymphocytes.

Predicted epitopes were assessed for multiple immune-relevant properties, as well as their ability to be accessed by a simulated antibody (antibody accessible surface area, AbASA). c) The final selected epitopes were linked together, along with an $\mathrm{N}$-terminal adjuvant, using EAAAK, GPGPG, AAY, and KK linkers. This sequence was assessed for immune-relevant properties and simulated immune response. d) The secondary and tertiary structures were predicted and refined, and the final 3D structure was docked using protein-protein docking to toll-like receptor 2 and 4 (TLR2/4). 


\section{RESULTS}

\section{Generation of Conformations for Linear B Lymphocyte Prediction}

Each of the simulated systems, including the 9 mutants, wild type, and the high mannose $\mathrm{N}$-glycan substituted wild type system, were assessed for stability along the entire 500 nanosecond simulation using the RMSD of all backbone atoms after least squares fitting to the same using standard GROMACS ${ }^{11}$ tools (Supplementary Figure 1). A total of $5 \mu \mathrm{s}$ of simulation time was used for linear B lymphocyte prediction. No system was deemed to have any stability issues, so each system was sampled at its initial conformation (after equilibration but before production dynamics simulation) and every 100 nanoseconds of simulation, yielding a total of 6 conformations for each of the 9 mutant and 1 wild type system. The high mannose system was not sampled in this way and was processed separately.

The high mannose system was further assessed for its antibody accessible surface area (AbASA). Using the built in GROMACS tool SASA, with a probe of size $0.72 \mathrm{~nm}$, the surface area was determined for the protein alone (Figure 2a, Supplementary Figure 2) while ignoring the glycosylation, and again while taking the glycosylation into account (Supplementary Figure 3). The percent change in the AbASA was determined as the change in the AbASA when taking the glycosylation into account (Figure 2b). This change in accessibility to an immune response was used to dictate which of the predicted epitopes would be included in COVCCF; an epitope was rejected if there was a large change in the AbASA due to the glycosylation, or if there were no residues with greater than $0.25 \AA^{2}$ of accessible surface area. Glycosylation of the spike glycoprotein creates 
a shield against immune response, reducing the AbASA for many surface residues by over $50 \%$, protecting otherwise targetable epitopes.
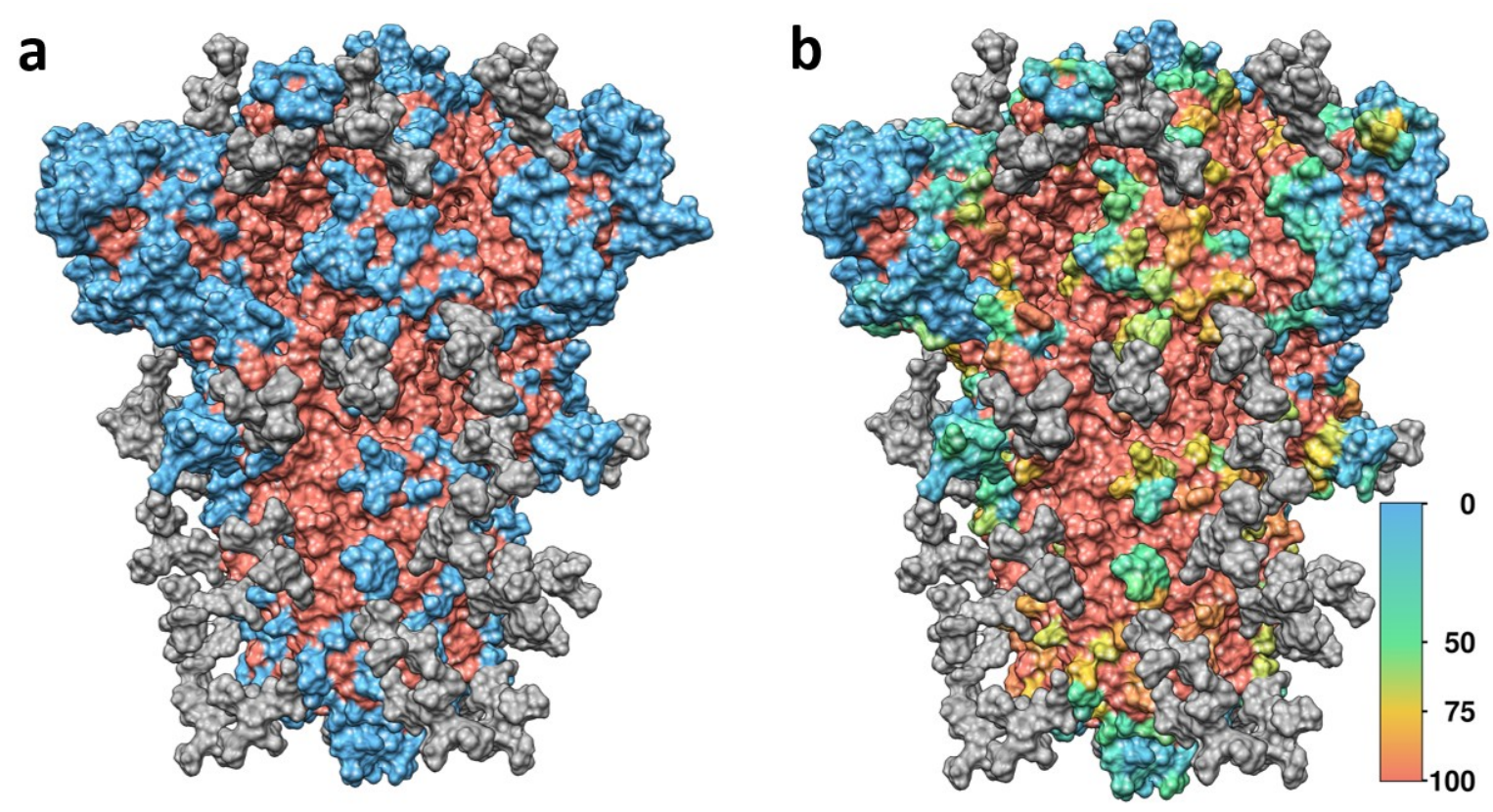

Figure 2: Antibody-accessible surface area (AbASA) of the spike glycoprotein. a) Regions of the spike glycoprotein which have at least $0.25 \AA^{2}$ of AbASA in blue, regions with less AbASA in red, and the glycosylation in gray. b) Percent change in AbASA due to glycosylation, with blue as no change, green as a $50 \%$ reduction in AbASA, and yellow a $75 \%$ reduction in AbASA. A value of $100 \%$ (red) was assigned for any residue with less than $0.25 \AA^{2}$ of AbASA.

\section{Identification of Antigenic Linear B-cell Epitopes}

ElliPro ${ }^{12}$ was used to predict the linear B-lymphocyte (LBL) epitopes for each of the 6 conformations for each of the nine mutant systems and the wild type. In total, this yielded 
3,311 epitopes, of which 428 unique epitopes were found (Supplementary Data 1). Sequences were tested for allergenicity, antigenicity, and toxicity; the sequences which passed these tests were then aligned to the full-length sequence of the viral protein to determine which of the epitopes did not have significant impedance due to the glycosylation. The LBL epitopes included in the final construct are given in Table 1. A total of 5 LBL epitopes were chosen for COVCCF.

Table 1: Predicted epitopes in the final vaccine construct.

\begin{tabular}{lll}
\hline LBL Epitope & HTL Epitope & CTL Epitope \\
& & \\
& FEYVSQPFLMDLEGK (1) & \\
& FNATRFASVYAWNRK (2) & SPRRARSVA (3) \\
NSNNLDSKVGGNYNYLY (4) & & SKVGGNYNY (4) \\
IYSKHTPINLVRDLPQGFSALELVDLPIG (5) & & \\
LPFFSNVTWFHAIHVSGTNGTKRFDNPVLPF (6) & LPFFSNVTWFHAIHV (6) & HVSGTNGTK (6) \\
& PFFSNVTWFHAIHVS (6) & VTWFHAIHV (6) \\
TPCNGVEGFNCYFPLQSYGFQPTNG (7) & NGVEGFNCYFPLQSY (7) & \\
& GVEGFNCYFPLQSYG (7) & \\
FQTLLALHRSYLTPGDSSSGWTAGAAAYY (8) & & WTAGAAAYY (8) \\
\hline
\end{tabular}

Note: Epitopes are numbered based on their order in the final vaccine construct. Epitopes with a shared sequence also share serial numbers; some LBL epitopes overlapped CTL/HTL epitopes, and some HTL epitopes overlapped CTL epitopes.

\section{Identification of Cytotoxic T Lymphocyte Epitopes}

Using all 10 sequences from the mutated and wild type proteins, a total of 3,844 nonunique epitopes were generated; 260 of these were unique (Supplementary Data 2). The 
epitopes which were predicted as immunogenic, antigenic, non-allergenic, and non-toxic were further assessed for their accessibility, yielding 6 total CTL epitopes (Table 1) included in the final construct. Epitopes which were either non-antigenic, allergenic, or toxic were not considered; accessibility was determined in the same fashion as for the LBL epitopes.

\section{Identification of Helper T Lymphocyte Epitopes}

As with the CTL prediction, all 10 sequences were submitted to the prediction server, with a total of 3,938 non-unique (and 272 unique) HTL epitopes (Supplementary Data 3). After predictions for their ability to induce cytokines, and assessment for antibody accessibility, $6 \mathrm{HTL}$ epitopes were included in the final vaccine (Table 1). Epitopes which did not elicit a response from IFN- $\gamma, \mathrm{IL}-4$, and IL-10 were not considered; accessibility was determined in the same fashion as for the LBL epitopes.

\section{Construction of the Multi-Epitope Vaccine Candidate}

In total, $5 \mathrm{LBL}, 6 \mathrm{HTL}$, and $6 \mathrm{CTL}$ epitopes were selected for the final multi-epitope vaccine candidate COVCCF. Epitopes for which there was overlap were not duplicated in the final construct, and were instead merged. The TLR4 agonist (UniProt accession ID P9WHE3), the 50 S ribosomal L7/L12 was added as an adjuvant on the $\mathrm{N}$-terminus of the construct, and linked to the vaccine peptide using an EAAAK linker. The GPGPG linker was chosen between the two HTL epitopes, an AAY linker between the HTL and CTL epitope, and KK linkers between the remaining epitopes. A $6 x$ His tag was added to the C-terminus to aid 
in purification. The final vaccine candidate consists of 331 amino acids and 9 separate linked protein sequences (Figure 3a, b).
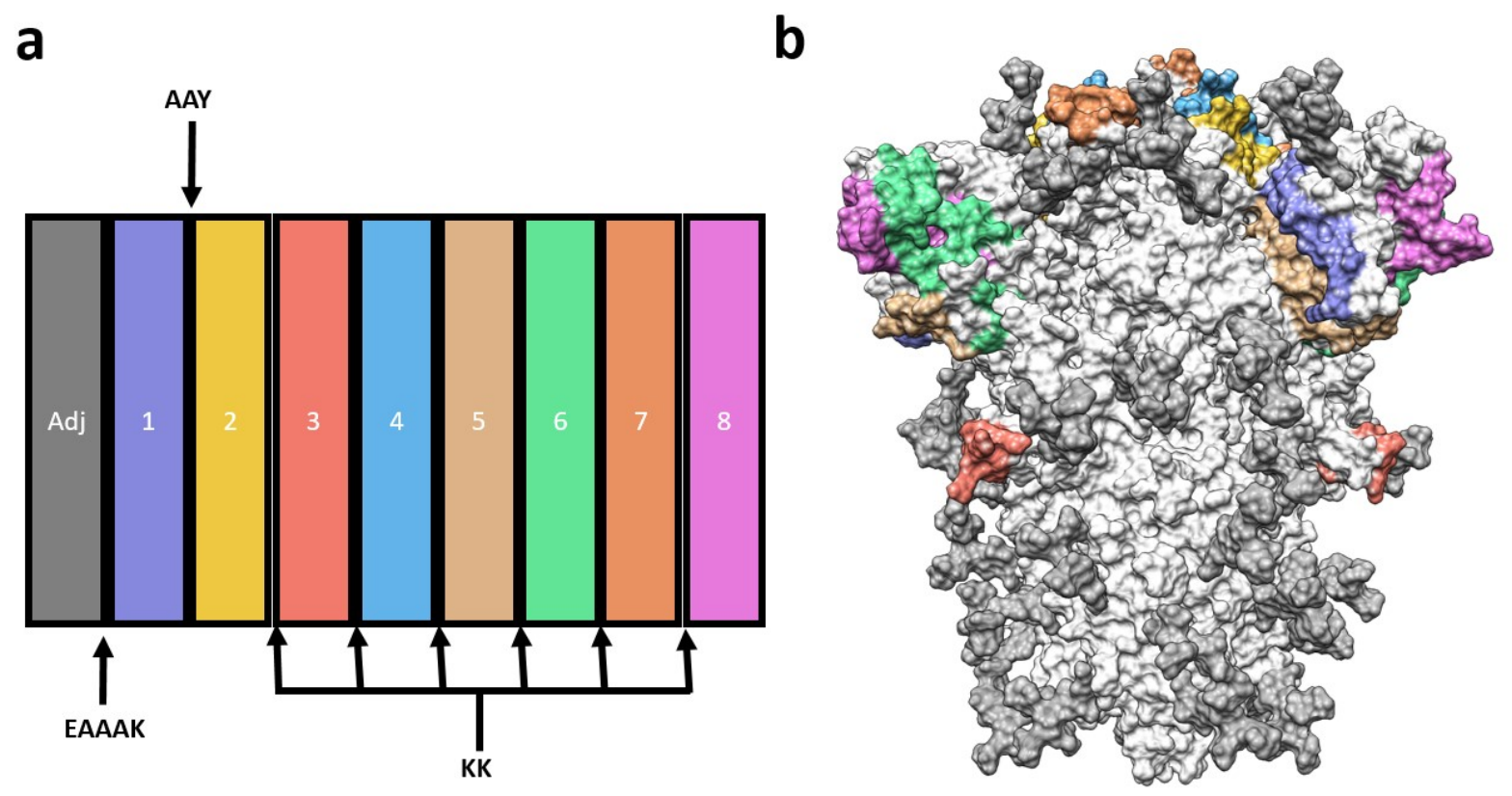

Figure 3: Epitope selection for the multi-epitope vaccine construct. a) Schematic representation of the final multi-epitope vaccine construct. Epitopes are labeled as in Table 1. b) Location of the selected epitopes on the spike glycoprotein. White residues indicate they are not in the final multi-epitope vaccine construct, with glycosylation in gray.

IFN-Y Inducing Epitope Prediction, Antigenicity and Allergenicity

A total of 323 IFN-y inducing epitopes were predicted using the scan function of the IFNepitope server ${ }^{13}$. Of these 323 predicted epitopes, 132 were predicted to have positive scores (Supplementary Data 4). These results are in line with the simulated 
levels predicted by C-ImmSim ${ }^{14}$ (Figure 4). The prediction for antigenicity from VaxiJen $2.0^{15}$ indicates it is antigenic under both a bacterial (0.5341) and viral model (0.4709) using the default threshold. COVCCF alone was also determined to be antigenic under both the bacterial $(0.6180)$ and viral $(0.5332)$ models using the default threshold of 0.4 . Both the full construct and the vaccine peptide without adjuvant were predicted as nonallergenic using AllerTOP 2.0 ${ }^{16}$. COVCCF is predicted to be non-allergenic, antigenic, and to elicit IFN- $\mathrm{y}$ induction.

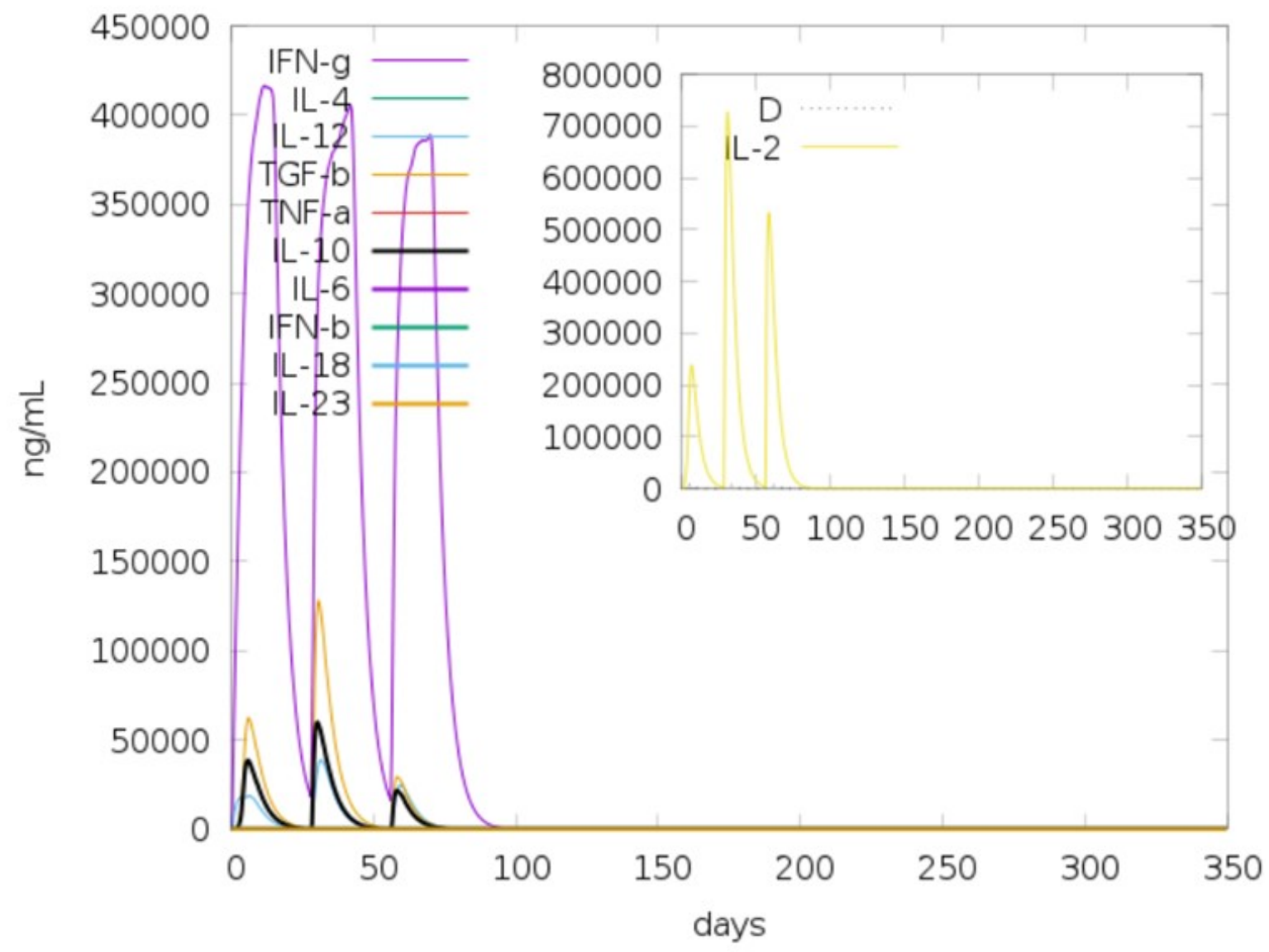

Figure 4: Cytokine levels induced by repeated injection of the vaccine construct. Levels were modeled in C-ImmSim based on three injections given 4 weeks apart. D in the inset plot is the danger signal (dotted line). 


\section{Physiochemical and Solubility Properties}

The physiochemical properties of COVCCF are outlined in Table 2. COVCCF is predicted to have a molecular weight of $35.9 \mathrm{kDa}$, with a theoretical isoelectric point of 8.75 , indicating a slightly basic protein. The half-life is predicted to be 30 hours in mammalian reticulocytes, $>20$ hours in yeast, and $>10$ hours in $E$. coli. The predicted instability index of 27.57 indicates a stable protein ( $>40$ indicates instability), while the aliphatic index of 79.09 indicates thermostability; a larger aliphatic index indicates higher stability. The predicted grand average of hydropathicity is -0.237 , which indicates the protein is hydrophilic; this value is calculated as an average over the entire protein of the hydropathicity of each amino acid, where hydrophilic amino acids have a negative value and hydrophobic amino acids have a positive value. The solubility score as determined by $\mathrm{CamSol}^{17}$ is 0.788 based on the sequence, with a corrected score of 1.994 . Altogether, COVCCF has ideal solubility and physiochemical properties.

Table 2: Predicted physiochemical properties of COVCCF.

\begin{tabular}{ll}
\hline Property & Result \\
\hline Amino acid count & 331 \\
Molecular weight & $35906.93 \mathrm{Da}$ \\
Chemical formula & $\mathrm{C}_{1633} \mathrm{H}_{2526} \mathrm{~N}_{432} \mathrm{O}_{471} \mathrm{~S}_{5}$ \\
Predicted pl & 8.75 \\
Estimated half-life: & \\
$\quad$ Mammalian reticulocytes & 30 hours \\
Yeast Cells & $>20$ hours \\
E. coli & $>10$ hours \\
Instability Index & 27.57 \\
Aliphatic Index & 79.89 \\
Grand average of hydropathicity (GRAVY) & -0.237 \\
Solubility & 1.994 \\
\hline
\end{tabular}




\section{Secondary Structure Prediction}

The final vaccine sequence was predicted to be $42.6 \%$ alpha helix, $9.4 \%$ beta sheet, and 48.0\% coil by PSIPRED $4.0^{18}$ (Supplementary Figure 4), while RaptorX property ${ }^{19}$ predicted $37 \%, 6 \%$, and $56 \%$, respectively. $57 \%$ of residues were predicted to be solvent exposed, $24 \%$ medium exposed, and $18 \%$ buried; a total of 58 residues (17\%) were predicted as disordered by RaptorX (Supplementary Figure 5). The secondary structure predictions were exported from PSIPRED for use in the tertiary structure modeling.

\section{Tertiary Structure Prediction, Refinement, and Validation}

Five models were predicted using the I-TASSER webserver ${ }^{20}$ based on alignments predicted by various threading programs. Z-scores for template alignments ranged from 0.84 to 5.61 , with $1 \mathrm{rquA}, 3 \mathrm{qtdA}, 1 \mathrm{dd} 3 \mathrm{~A}, 1 \mathrm{dd} 4 \mathrm{~A}$, and $2 \mathrm{ftc}$ as the top 5 ranking templates. Model 1 (Figure 5a) was selected for further refinement. A local installation of ModRefiner ${ }^{21}$ was used for the initial refinement of the model in the two-step process outlined in the methods. The model refined using ModRefiner was then submitted to a local installation of GalaxyRefine ${ }^{22}$, where 10 models were generated for further assessment. The ERRAT server was used to assess the generated model, with model 1 (Figure 5b) having the highest quality factor of 81.013. Furthermore, ProSA-web ${ }^{23}$ was additionally used for validation, indicating a Z-score of -7.41 , well within the range of native proteins of comparable size (Figure 5c). The Ramachandran plot indicated $92.7 \%$ of residues were in favored regions, $99.4 \%$ were in favored or allowed regions, and only 
two residues were in outlier regions (Figure $\mathbf{5 d}$ ). These results indicate our predicted structure is likely to be close to the actual 3D structure.
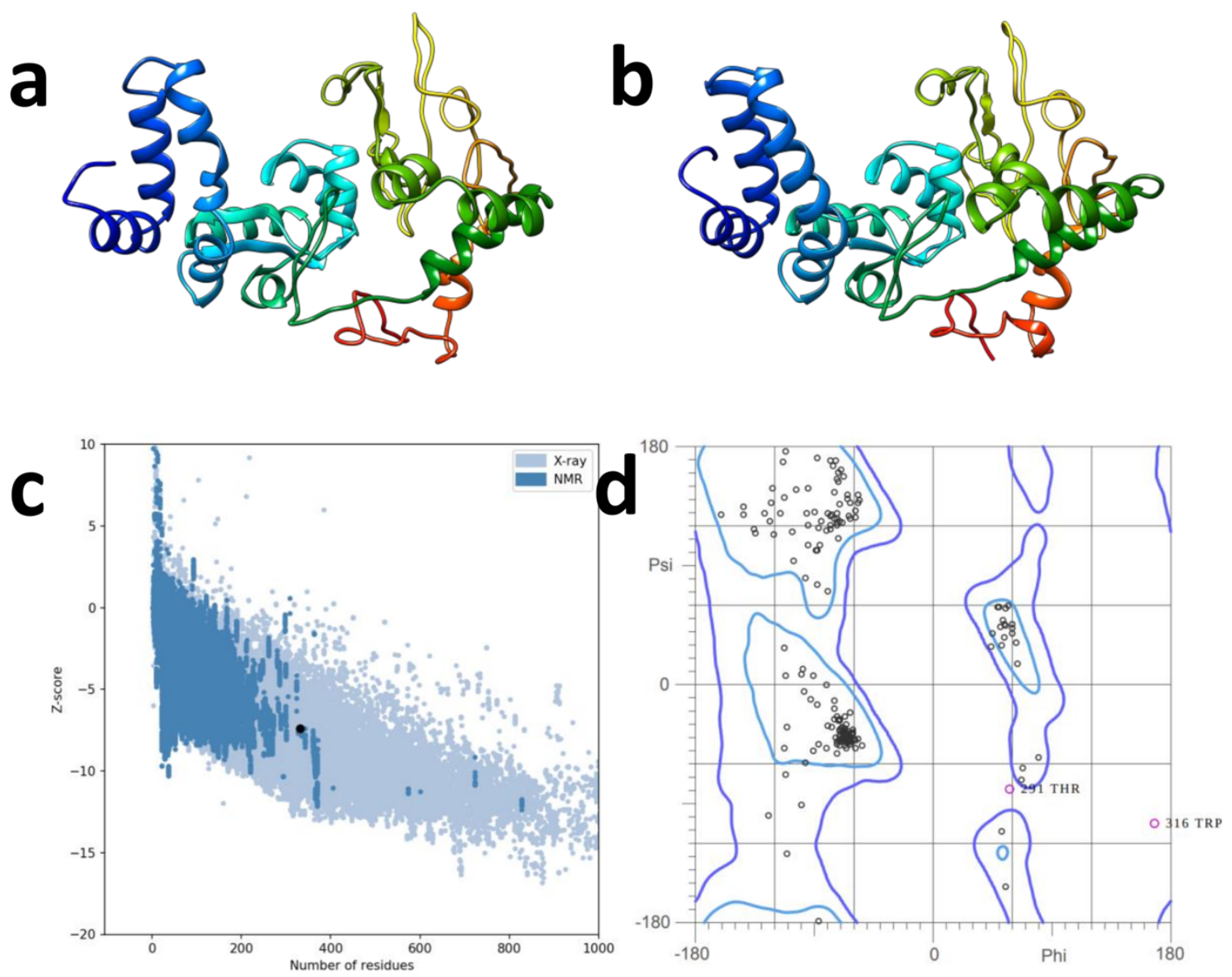

Figure 5: Construction and refinement of the multi-epitope vaccine construct. a)

Final 3D model of the vaccine construct after modeling with I-TASSER. b) Refined model after refinement with ModRefiner and GalaxyRefine. c) Validation of structure with ProSA-web, indicating the structural properties are in line with other proteins of similar size (Z-score -7.41). d) Ramachandran plot indicating $92.7 \%$ of residues are in favored regions, and 2 residues are in outlier regions. 


\section{Prediction of LBL Epitopes in Final Vaccine Construct}

A total of 8 discontinuous and 13 linear epitopes were found in COVCCF. The discontinuous epitopes ranged in length from 10 to 46 amino acids, encompassing a total of 213 of the 331 residues in the protein construct. The 13 linear epitopes were nonoverlapping and encompassed 186 residues. Scores ranged from 0.508 to 0.832 for the linear epitopes, and 0.558 to 0.809 for the discontinuous epitopes. This result indicated COVCCF has the ability to induce an immune response not only from the selected epitopes, but from conformational discontinuous epitopes based on its 3D structure.

\section{Protein-Protein Docking to TLR2 and TLR4}

Protein-protein docking was performed using the HADDOCK 2.4 webserver ${ }^{24}$ with a datadriven approach. First, CPORT ${ }^{25}$ was implemented to determine the predicted residues in a protein-protein interaction. Residues from the adjuvant were selected as part of the interaction with both toll-like receptors, since it has been shown as able to induce an immune response ${ }^{26}$. In the vaccine, residues F32, V34, T35, A36, A38, P39, V42, A43, A45, G46, A48, P49, and A50 were selected to drive the docking, while in the adjuvant alone residues $\mathrm{T} 35, \mathrm{~A} 36, \mathrm{~A} 38, \mathrm{P} 39, \mathrm{~A} 41, \mathrm{~V} 42, \mathrm{~A} 43, \mathrm{~A} 45, \mathrm{G} 46, \mathrm{~A} 47$, and P49 were chosen. In TLR4, residues 148, D50, N51, L52, P53, F54, S55, D60, P65, H68, G70, S71, Y72, S73, F75, S76, P78, D84, S86, D95, Q99, and S100 were chosen, and residues R63, T65, S85, G87, Y109, Y111 were chosen for TLR2. CPORT predicted many more residues as active, but they were not chosen in an effort to narrow the results (Supplementary Data 5). Surrounding residues were not entered as input in HADDOCK; 
instead, the default selection for passive residue selection was used, defining a 6.5 angstrom radius around active residues as the passive region.
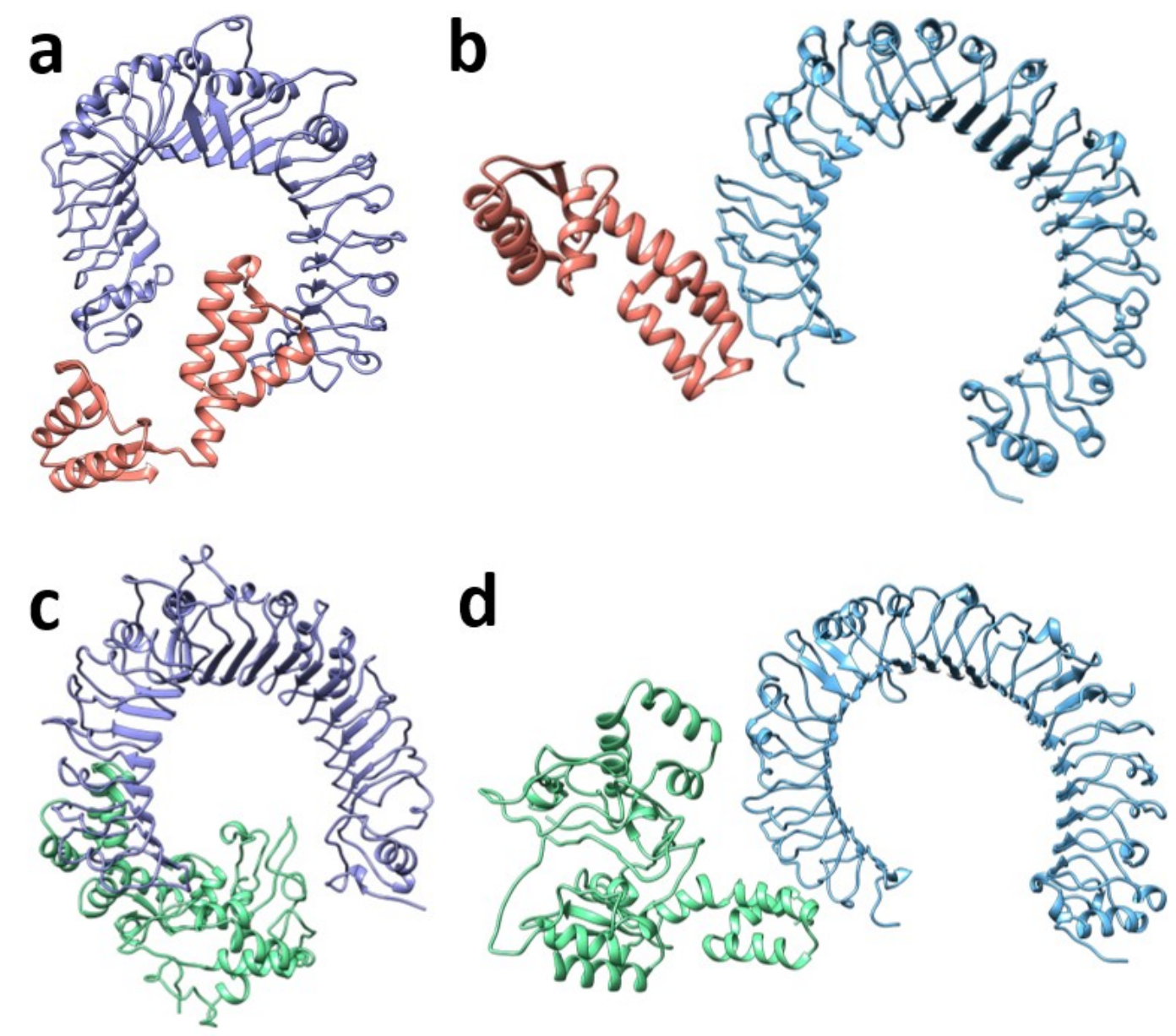

Figure 6: Protein-protein docking results of adjuvant or vaccine construct with

TLR2 or TLR4. Results from HADDOCK for the a) adjuvant and TLR2, b) adjuvant and TLR4, c) vaccine and TLR2, and d) vaccine and TLR4.

The predicted scores for each of the resulting structures (Figure 6a-d) are outlined in Table 3. The best predicted binding poses for both the TLR2 and TLR4 constructs indicated the vaccine construct induces a conformational change in the adjuvant region which is beneficial to the interaction; the $K_{d}$ for the vaccine-TLR complexes is 
comparatively lower in both cases. This is likely due to a predicted increase in the number of interfacial contacts between the complexes. For example, in the TLR4 complexes, four of the six interfacial contact types increased (charged-charged from 3 to 4 , charged-polar from 5 to 10, charged-apolar from 5 to 10, and apolar-apolar from 23 to 27), one remained the same (polar-polar at 2) and one decreased (polar-apolar from 13 to 12). Altogether, the docking results indicate that COVCCF will be able to bind TLR2/TLR4 and induce an immune response.

Table 3: Results from protein-protein docking in HADDOCK and binding affinity predictions in PRODIGY.

\begin{tabular}{r|llll}
\hline \multicolumn{1}{c}{$\begin{array}{l}\text { Cluster Score } \\
(\mathbf{\pm})\end{array}$} & Z-Score & $\begin{array}{l}\text { Best } \\
\text { Structure } \\
\text { Score }\end{array}$ & $\begin{array}{l}\text { PRODIGY K } \\
\text { Prediction }\end{array}$ \\
\hline $\begin{array}{r}\text { Adjuvant - } \\
\text { TLR2 }\end{array}$ & $-76.7(5.0)$ & -1.4 & -83.422 & $1.3 \mathrm{E}-07$ \\
$\begin{array}{r}\text { Vaccine - } \\
\text { TLR2 }\end{array}$ & $-84.6(1.4)$ & -1.7 & -86.138 & $3.3 \mathrm{E}-08$ \\
$\begin{array}{r}\text { Adjuvant - } \\
\text { TLR4 }\end{array}$ & $-81.7(2.9)$ & -1.4 & -85.447 & $2.6 \mathrm{E}-07$ \\
$\begin{array}{r}\text { Vaccine - } \\
\text { TLR4 }\end{array}$ & $-84.3(2.3)$ & -1.9 & -87.720 & $2.2 \mathrm{E}-07$ \\
\hline
\end{tabular}

Note: The cluster score and Z-score are the aggregate scores for all proteins within the best cluster, while the best structure score is for the structure with the lowest HADDOCK score. The PRODIGY prediction is for the predicted best structure by HADDOCK score. TLR: toll-like receptor.

\section{In Silico Codon Optimization}

The Java Codon Adaptation Tool ${ }^{27}$ was used to optimize codon usage of the vaccine construct, to be expressed in E. coli (K12). This optimization would allow for maximal protein expression. A 993 base pair sequence was generated with a Codon Adaptation 
Index (CAI) value of 0.916 , and a GC content of $50.25 \%$, which compares favorably with the $50.73 \%$ GC content in the chosen E. coli strain. The sequence of the recombinant plasmid was then inserted in a pET30a (+) vector using SnapGene software (www.snapgene.com, Figure 7). These results indicate our protein would likely be easily cloneable in a common vector, the $\mathrm{K} 12$ strain of $E$. coli.

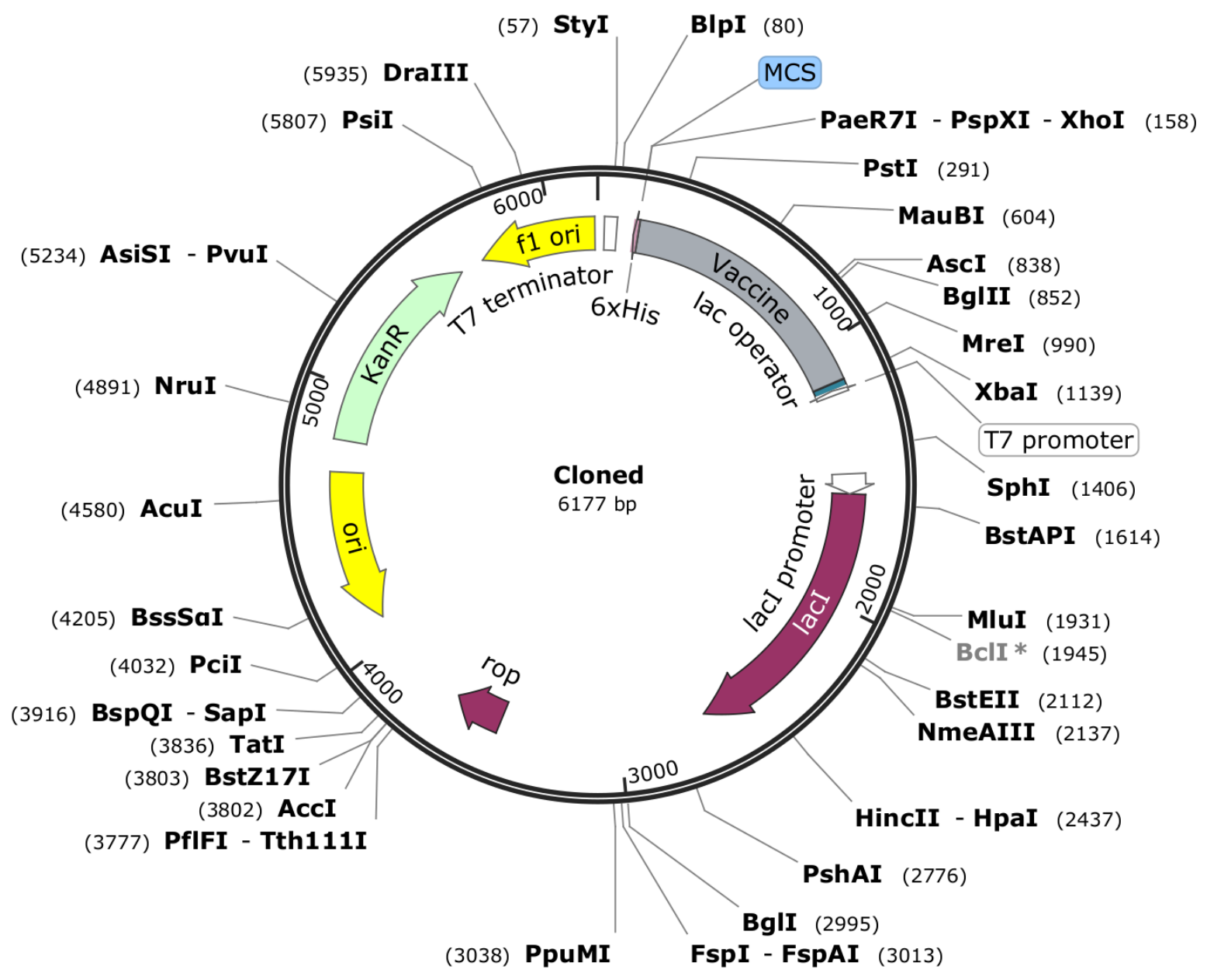

Figure 7: In silico cloning of the vaccine construct using the pET30a (+) expression vector. The vaccine insertion is denoted with a gray bar. The His-tag is located at the C-terminal end. MCS: multiple cloning site; KanR: kanamycin resistance protein. 

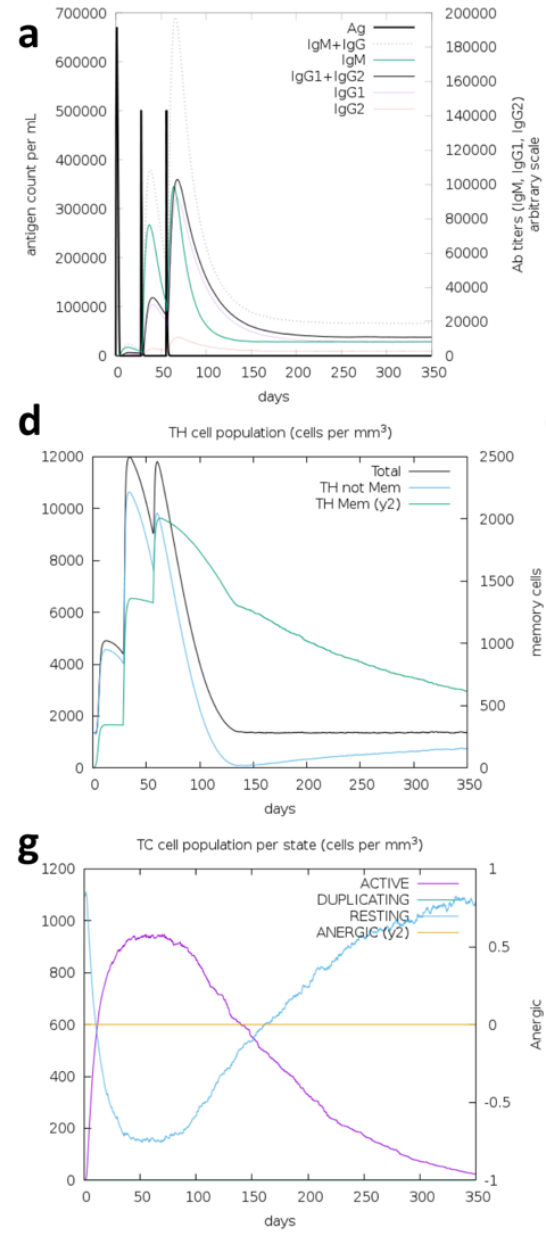

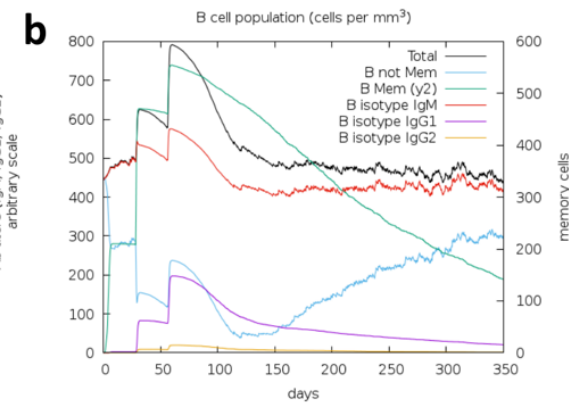

e

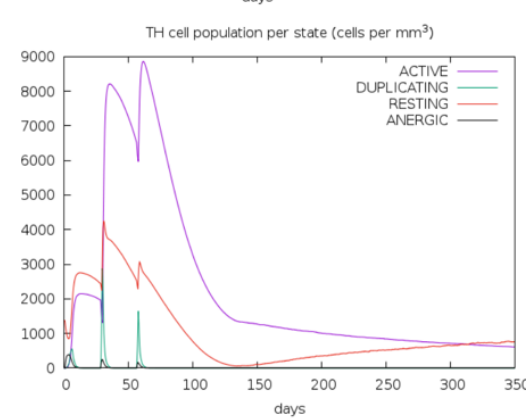

h

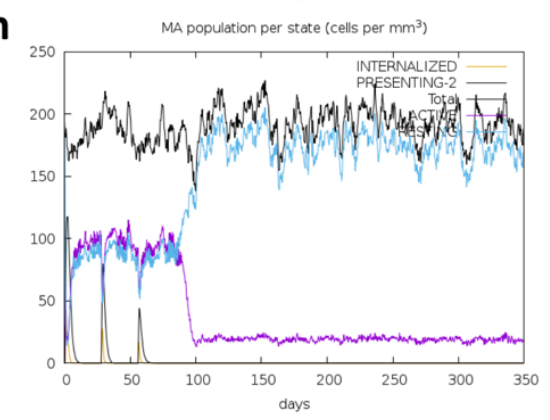

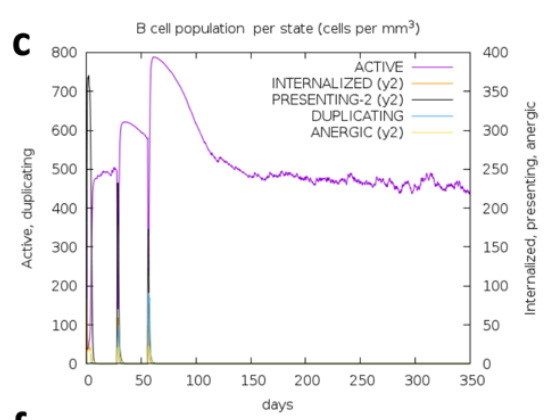

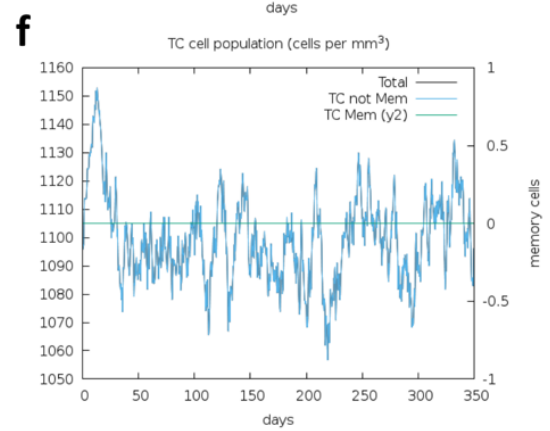

i

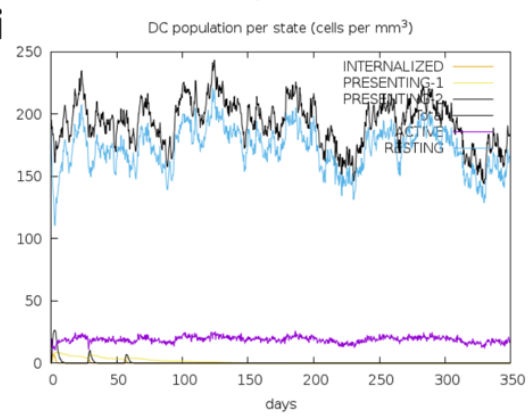

Figure 8: Immune simulation results from C-ImmSim. Injections were simulated to occur at $\mathrm{T}=0,4$, and 8 weeks. a) Antigen and immunoglobulin levels, subdivided per isotype; B lymphocytes by b) total count and c) population per entity state (active, presenting, internalized, duplicating, or anergic); CD4 T-helper lymphocytes by d) count and e) population per entity state; CD8 T-cytotoxic lymphocytes by f) count and g) population per entity state; total count of $h$ ) macrophages and i) dendritic cells. Supplementary Figures 6-14 include high-resolution copies of these figures.

\section{Immune Simulation Indicates Strong Secondary and Tertiary Response}

The immune simulations carried out on the $\mathrm{C}-\mathrm{ImmSim}^{14}$ server gave results consistent with an actual immune response, highlighted by the increased secondary response when 
compared to the primary response. High levels of immunoglobulin activity (IgM, IgG1, $\lg G 2)$ in the secondary and tertiary response were matched with a corresponding decrease in antigen concentration (Ag, Figure 8a). B-cell population also increased with each injection (Figure $\mathbf{8 b}, \mathbf{c}$ ), while a corresponding T-helper and cytotoxic T-cell response was evident as well (Figure $\mathbf{8 d} \mathbf{d} \mathbf{g}$ ). Additionally, macrophage activity was increased, while dendritic cell activity remained consistent during exposure (Figure $8 \mathbf{h} \mathbf{i}$ ). Taken together, these results support the immunogenicity of COVCCF.

\section{DISCUSSION}

While there are currently numerous drug candidates and vaccine candidate undergoing clinical trials, there is yet to be a proven effective treatment or cure for COVID-19, the disease caused by SARS-CoV-2. As of June $23^{\text {rd }}$, the 7-day moving average for cases is pushing toward 150,000 per day; it is clear that further work toward the discovery of a vaccine must be done. Here, we have used computational techniques, including molecular dynamics simulations and immunoinformatics techniques, to design a multiepitope vaccine candidate which appears capable of eliciting an immune response. The full multi-epitope vaccine sequence was predicted to contain 132 IFN- $\gamma$ positive epitopes; this line up well with the predicted induction of IFN-y from C-ImmSim, which predicted levels over $400,000 \mathrm{ng} / \mathrm{mL}$ for both the primary and secondary doses (Figure 3). The immune simulation indicated results consistent with an expected immune response to a vaccine, based on the general increase in the immune response upon secondary and tertiary doses of vaccine. Protein-protein docking comparisons between the full vaccine 
construct with the adjuvant alone indicated a stronger interaction with both TLR2 and TLR4 in the vaccine than the adjuvant alone, indicating a potential shift in conformation which allows for better binding. This stronger interaction could indicate a quicker immune response to the vaccine candidate.

Key to this work was the implementation of the glycan shield for the selection of epitopes to be included in the final vaccine construct COVCCF. We believe it to be important to design a vaccine which would be capable of creating an immune response which would be effective against the SARS-CoV-2 spike glycoprotein in both its unglycosylated and fully glycosylated states; this unfortunately means not including epitopes which, though they may elicit a strong immune response, would generate antibodies which would not be capable of reaching their intended target. An example of this is a predicted LBL epitope from A701 through 1720 , predicted in all nine mutant systems and the wild type. However, while there are residues in this region which have some antibody-accessible surface area in a non-glycosylated protein, glycosylation of residues N709 and N1074 almost completely abolish this accessibility. As an example, S704 has $11.4 \AA^{2}$ of AbASA when glycosylation is not accounted for (using a probe size of $0.72 \mathrm{~nm}$ ), but is reduced to $1.04 \AA^{2}$ of AbASA when glycosylation is accounted for. While there is a limitation to blindly following the probe size of $0.72 \mathrm{~nm}$ as the only criteria, the knowledge that this probe size only accounts for an averaged loop radius, and not the size of the loop-containing antibody, was taken into account when selecting epitopes where some interference from glycosylation was evident.

In addition, as opposed to taking a sequence-based approach to the prediction of LBL epitopes, we chose to include conformational changes which could uncover more 
epitopes, which may not be found using only the amino acid sequence. Additionally, we included multiple mutated systems, with the hopes of improving our chances of discovering epitopes which may not be discovered when using only the wild-type structure. To do this, we used 500 nanosecond molecular dynamics simulations of 10 different systems, which included 9 mutated systems and the wild-type system. This expanded our predictions in more than one way; for example, the initial equilibrated system which was to be submitted to unrestrained molecular dynamics simulation generated 51 LBL epitopes, while a combination of this conformation with conformations at $100,200,300,400$, and 500 nanoseconds of simulation yielded 120 unique LBL epitopes. Further adding to our predictions, the 9 mutated systems added another 309 unique LBL epitopes not predicted in the 6 conformations used for the wild-type system. In fact, only one out of the five LBL epitopes included in the final vaccine construct was predicted in any of the conformations of the wild-type system. It should be noted that although each of the 10 systems was used for predictions of CTL and HTL epitopes as well, since only the primary structure of the protein is used for these predictions, it was not expected that there would be a significant increase in the number of epitopes due to the inclusion of the mutants.

The final multi-epitope vaccine construct (COVCCF) consisted of antigenic, nontoxic, non-allergenic, and antibody accessible B-cell and T-cell epitopes; in addition, multiple helper T-cell epitopes, all of which were determined to induce cytokines important to innate immunity, such as IFN- $y$, IL4, and IL10, were included. Our $35.9 \mathrm{kDa}$ protein was predicted to be soluble upon overexpression in an E. coli host, with a theoretical pl of 8.75 , implying its best stability would be in a slightly basic environment. The instability 
index indicates a protein that is likely to be stable in a test tube; a protein with an instability index (II) greater than 40 is not predicted to be stable, whereas the II of COVCCF is 27.57. Additionally, the aliphatic index is a positive factor for the increase of thermostability, for which our vaccine construct was scored at 79.09. Finally, the negative value for the grand average of hydropathy (GRAVY), -0.237 , indicates a hydrophilic protein, allowing it to properly interact with water molecules. The in vivo half-life was predicted using the "Nend rule"; the "N-end rule" relates the half-life of a protein to the identity of the $\mathrm{N}$-terminal residue, which for this protein is a methionine. Outside of an $\mathrm{N}$-terminal valine, this yields the highest predicted half-life for the vaccine construct, which is a measure of how long it would take for half of the amount of protein in the cell to disappear, based on host.

Further functional validation of the final multi-epitope vaccine candidate would be required prior to implementation, the first step of which is screening for immunoreactivity using serological analysis ${ }^{28}$. Expression of the protein in a suitable host would be required for this, with $E$. coli the preferred choice for recombinant protein expression. Both the value for the codon adaptation index (0.916) and GC content $(50.25 \%)$ were favorable, indicating the probability of high expression in the selected E. coli (strain K12) vector. Validation of effectiveness in an animal model is also a possibility, with studies in ferrets indicating rapid transmission and indicate efficacy in identification of therapeutic applications 29,30 .

A key limitation to this study is the lack of inclusion of mutants in the SARS-CoV-2 spike glycoprotein which have proven to have much higher prevalence in the general population. Specifically, the D614G mutation, which in certain locations has become the predominant species ${ }^{31}$, was not included. Selection of mutants for simulation studies was 
completed before the prevalence of this mutation had been demonstrated. It is not known if this would impact the selection of LBL epitopes for the final candidate, though CTL and HTL epitope selection would largely be unaffected since both are sequence based as opposed to conformation based in this study.

It is becoming clear that control of SARS-CoV-2, the virus which causes COVID19, will require a vaccine in order to generate enough herd immunity to prevent overwhelming the capabilities of hospitals around the world. Our integration of molecular dynamics simulations and immunoinformatics techniques has allowed us to generate a potential multi-epitope vaccine, coding for multiple LBL, CTL, and HTL epitopes. The inclusion of 9 mutated spike glycoproteins, along with long timescale molecular dynamics simulations, allowed for greater sampling of conformations for the spike glycoprotein; this sampling improved our ability to select LBL epitopes which are immunogenic, antigenic, and are not shrouded by the glycan shield. The final 331 amino acid peptide vaccine, COVCCF, with its ideal physiochemical properties and ability to initiate an immune response, could be a first step in preventing the further spread of COVID-19. If broadly applied, the synergistic computational approaches presented here can be utilized to design vaccines for other emerging infectious diseases as well.

\section{METHODS}

\section{System Selection}

Prior to epitope prediction, a total of 10 systems were simulated using the GROMACS $2020.1^{11}$ molecular dynamics package, including 9 mutated systems and wild type. The 
selected mutants (A852V, A930V, F797C, F970S, L752F, L861M, V615L, V860D, V860L) were selected ${ }^{32}$ based on conservation of the residue between SARS-CoV-2, SARS-CoV1, and MERS-CoV. Each of the 9 mutations were added in silico using CHARMM-GUI ${ }^{33,34}$ using PDB ID $6 \mathrm{VSB}^{35}$ at the time of system creation. Following a processing step involving the addition of hydrogen atoms and completion of missing loops, a water box with edges at least 10 angstroms from any part of the protein was added. The systems were neutralized and brought to a total ionic strength of $0.15 \mathrm{M}$ using sodium and chloride ions. Parameterization of the protein, ions, and TIP3P water molecules was accomplished using the CHARMM36m force field ${ }^{36}$. Each of these systems used the glycosylation state as in the crystal structure with no modifications. An additional wild type system with high mannose $\mathrm{N}$-glycans was constructed in order to assess the change in the proteins immune accessibility due to the glycan shield.

\section{Simulation Parameters}

All systems were simulated using GROMACS 2020.1 on the AiMOS Supercomputer at the Rensselaer Polytechnic Institute Center for Computational Innovations in a three-step process. Initial minimization of the systems was run until changes in the potential energy of the system reached machine precision. Following minimization, an NVT equilibration step was completed with a 2 fs timestep for 500,000 steps using $400 \mathrm{~kJ} \mathrm{~mol}^{-1} \mathrm{~nm}^{-2}$ and $40 \mathrm{~kJ} \mathrm{~mol}^{-1} \mathrm{~nm}^{-2}$ positional restraints on the backbone and sidechains, respectively. A 500 ns production step was completed using the NPT ensemble with no position restraints and a 2 fs timestep. 
Hydrogen atoms were constrained using the LINCS $^{37}$ algorithm. Temperature for the system was held at $300 \mathrm{~K}$ using a Nose-Hoover thermostat ${ }^{38}$ with a 1 ps coupling constant. For the production simulation, pressure was coupled isotropically using a Parrinello-Rahman barostat ${ }^{39}$ with a 5.0 ps coupling constant and compressibility of $4.5 \mathrm{e}-$ 05 bar $^{-1}$ to maintain a pressure of 1 bar. The pair-list cutoff was constructed using the Verlet scheme ${ }^{40}$ with a cutoff distance of $1.2 \mathrm{~nm}$. Particle mesh Ewald electrostatics ${ }^{41}$ were used to describe coulombic interactions with a $1.2 \mathrm{~nm}$ cutoff, while van der Waals forces were smoothly switched to using between 1.0 and $1.2 \mathrm{~nm}$ using a force-switch modifier to the cut-off scheme. Linear center of mass translation was removed every 100 steps for the entire system.

\section{Antibody-Accessible Surface Area Determination}

To determine which predicted epitopes are most likely to be capable of eliciting a useful immune response, we determined the antibody-accessible surface area (AbASA) using a method similar to that outlined previously ${ }^{42}$. Two calculations for AbASA were completed using the built in SASA tool in GROMACS 2020.1, selecting a probe size of 0.72 nanometers as opposed to the standard 0.14 nanometer probe used for a standard SASA calculation. The first calculation determined the AbASA for the bare protein, not accounting for glycosylation, while the second determined the AbASA for the protein while also taking the extensive glycosylation into account. When selecting an epitope for COVCCF, a residue was deemed to be not antibody accessible if its AbASA was lower than $0.25 \AA^{2}$. As the spike glycoprotein is a homotrimer, an average of the AbASA across the three domains was used for this determination. Additionally, residues with a drop in 
AbASA when considering the glycan shield were inspected on a case-by-case basis with the knowledge that the $0.72 \mathrm{~nm}$ probe radius would only account for accessibility for an average loop in an antibody, and did not account for accessibility of an entire antibody. Regions which had a large change in AbASA were determined to be shielded, and predicted epitopes for these regions were not included in COVCCF.

\section{Linear B-cell Epitope Prediction}

The above simulations were sampled at $t=0,100,200,300,400$, and 500 nanoseconds

for the prediction of linear B lymphocyte (LBL) epitopes, yielding a total of 60 structures. These structure-based predictions were made using ElliPro ${ }^{12}$ using the default minimum score of 0.5 and the default maximum distance of 6 angstroms. ElliPro implements three algorithms in its predictions: 1) an approximation of the shape of the protein as an ellipsoid; 2) calculation of the protrusion index for each residue, which is a quantification of the extent to which a residue protrudes from the surface of a protein based on the ellipsoid approximations; and 3) a clustering of neighboring residues based on protrusion index. While ElliPro is able to predict both linear and conformational epitopes, only linear epitopes are used in vaccine design ${ }^{43}$. Since only structural epitopes were generated, only residues 27 through 1146 were included in any of the epitope predictions, as those are the only residues crystallized in the pdb used.

\section{Cytotoxic T Lymphocytes (CTL) Epitope Prediction}

Cytotoxic $T$ lymphocyte (CTL) epitopes were predicted for each of the 10 sequences noted above using the NetCTL 1.2 server $^{44}$. The default threshold for epitopes was 
retained at 0.75 , and all 12 of the available MHC class I supertypes was used for the predictions. NetCTL uses artificial neural networks to predict major histocompatibility class $(\mathrm{MHC})$ I binding and proteasomal cleavage, while TAP transport efficiency is predicted using a weight matrix. Additionally, the CTL epitopes were each evaluated for their immunogenicity using the MHC-1 immunogenicity tool on the IEDB server ${ }^{45}$.

\section{Helper T Lymphocytes (HTL) Epitope Prediction}

Helper T cells help activate B cells to secrete antibodies and macrophages, and also help activate cytotoxic $T$ cells, indicating their importance to adaptive immunity. Prediction of these HTL epitopes as peptides that bind MHC II molecules is therefore key to rational vaccine design ${ }^{46}$. HTL epitopes of length 15 were predicted using the IEDB MHC-II binding predictions tool. The IEDB recommended prediction method was selected, which uses the consensus approach ${ }^{47}$, combining NN-align ${ }^{48}, \mathrm{SMM}^{-a l i g n}{ }^{46}, \mathrm{CombLib}^{49}$, and Sturniolo ${ }^{50}$ when possible, otherwise using NetMHCIlpan ${ }^{51}$. The full HLA reference set was used for the prediction, and predictions with a percentile rank $\leq 2$ were chosen; a lower percentile rank indicates a higher affinity.

\section{Assessment of CTL/LBL Epitopes for Antigenicity, Allergenicity, and Toxicity}

To ensure their ability to illicit an immune response, the antigenicity of each of the CTL and LBL epitopes was evaluated using the VaxiJen 2.0 server $^{15}$. VaxiJen uses an alignment-free approach based on auto cross covariance (ACC) transformation, a protein sequence mining method which has been applied to quantitative structure-activity relationships (QSAR) studies and protein classification ${ }^{52}$. This application of ACC to the 
principal component analysis (PCA) of 29 properties of each of the 20 amino acids allows for the removal of irrelevant information, amplifying class-discriminating properties. Allergenicity of epitopes was determined using the AllerTOP 2.0 server $^{16}$, which in addition to ACC uses a k-nearest neighbor algorithm based on a training set consisting of 2427 each of known allergens and non-allergens from different species. Toxicity of epitopes was predicted using the ToxinPred ${ }^{53}$ server, which uses the Support Vector Machine (SVM) algorithm, with a main dataset including 1805 sequences as positive training data and 3593 negative sequences from Swissprot ${ }^{54}$, and an independent dataset comprising of 303 positive and 300 negative sequences, also from Swissprot.

\section{Identification of Cytokine-Inducing HTL epitopes}

The ability of an HTL epitope to induce cytokines (specifically, interferon-gamma [IFN- $\mathrm{Y}]$, interleukin-4 [IL-4], and interleukin-10 [IL-10]) is key to a vaccine's ability to illicit an effective immune response; the release of these cytokines helps in the activation of cytotoxic T-cells and other immune cells ${ }^{13}$. To determine the ability of our predicted HTL epitopes to induce these cytokines, we used the IFNepitope server ${ }^{13}$ (IFN-y) using the motif and SVM hybrid approach with the IFN-gamma versus non IFN-gamma model; IL4pred server ${ }^{55}$ (IL-4) using the hybrid (SVM + motif) and the default SVM threshold of 0.2; and IL10pred server ${ }^{56}$ (IL-10) using the SVM based method with the default SVM threshold of -0.3 . These prediction servers, like ToxinPred above, use the SVM algorithm for their predictions. 


\section{Construction of the Multi-Epitope Vaccine Candidate Sequence}

The CTL, HTL, and LBL epitopes which passed the above tests were used to generate the full vaccine sequence. In the event of overlap between epitopes, we did not duplicate the sequence. Epitopes were linked together using GPGPG, AAY, and KK linkers; GPGPG and AAY linkers were used to connect the HTL and CTL epitopes, while KK linkers were used for B-cell epitopes, allowing them to preserve their independent immunogenic properties ${ }^{57}$. A $50 \mathrm{~S}$ ribosomal protein L7/L12 (locus RL7_MYCTU, UniProtKB ID: P9WHE3) was chosen as an adjuvant, and inserted on the $\mathrm{N}$-terminus using an EAAAK linker.

\section{Prediction of Physiochemical Properties, Solubility, Allergenicity, Antigenicity, and}

\section{IFN-y induction}

The full sequence for the multi-epitope vaccine construct COVCCF was tested for its allergenicity using the AllerTOP 2.0 server, and its antigenicity using the VaxiJen 2.0 server. The IFNepitope server was used to scan the full sequence for predicted IFN-Y inducing epitopes using the SVM based method for score prediction only. The ProtParam web server ${ }^{58}$ allows for the prediction of various physiochemical properties, including amino acid composition, theoretical isoelectric point (pl), instability index, half-life (both in vivo and in vitro) aliphatic index, molecular weight, and grand average of hydropathicity (GRAVY). Solubility of the final protein sequence was predicted using the CamSol server ${ }^{17,59}$, which allows for a pdb structure as input, taking into account the $3 \mathrm{D}$ conformation of the protein as opposed to only the sequence. 


\section{Prediction of Secondary Structure}

The generated sequence for the full-length vaccine was submitted to the PSIPRED 4.0 server ${ }^{18}$ to predict its secondary structure. PSIPRED uses a deep neural network architecture with two hidden layers and rectifier activations; the current version has a Q3 secondary structure prediction accuracy of $84.2 \%$. The RaptorX Property server ${ }^{19}$ was additionally employed as a second validation. RaptorX Property employs a new machine learning model, Deep Convolutional Neural Fields (DeepCNF), which implements both conditional neural fields (CNF) and deep convolutional neural networks (DCNN).

\section{Tertiary Structure Prediction}

Homology modeling of the final vaccine candidate was completed using the I-TASSER server $^{20}$. I-TASSER (Iterative Threading ASSEmbly Refinement) uses a three-step process to model the tertiary structure of a protein. First, the server tries to retrieve template proteins from the PDB library using LOMETS (Local Meta-Threading Server), which generates protein structure predictions by ranking and selecting models from multiple state of the art threading programs ${ }^{60}$. The second step involves assembling fragments excised from the PDB templates determined in step 1 using replica-exchange Monte Carlo simulations, with unaligned regions generated using ab initio modeling. The third step integrates spatial restraints to remove steric clashes, finally generating full atomic models. The secondary structure predictions generated by PSIPRED were submitted along with the primary structure of the multi-epitope vaccine candidate. 


\section{Tertiary Structure Refinement}

The selected model generated by I-TASSER was further refined first using ModRefiner ${ }^{21}$, followed by GalaxyRefine ${ }^{22}$. ModRefiner uses a two-step process, first a low-resolution step followed by a high-resolution step. The low-resolution step begins with a C-alpha trace of the initial structure, adding main chain atoms for an energy minimization. This structure is then passed to the high-resolution step, which adds side chain atoms and does a full atomic energy minimization, yielding a final model. GalaxyRefine begins by rebuilding all side chains, placing the highest probability rotamers starting from the core and extending to the surface, layer by layer. Upon reaching a steric clash, the next highest probability rotamer is selected. After being rebuilt, the model is refined using a two-step relaxation process, of which the lowest energy model is returned as model 1 , and additional models are returned based on the closest clustered models.

\section{Structural Validation}

Multiple servers were implemented to validate the tertiary structure generated. ProSAweb $^{23}$ gives an assessment of the overall model quality, displayed in the context of all Xray and NMR structures, with a Z-score falling outside that of known structures generally implies errors in the structure. The ERRAT server ${ }^{61}$ was used to assess non-bonded interactions in comparison to high-resolution crystallographic structures. Finally, the MolProbity ${ }^{62}$ server was used to generate a Ramachandran plot, which gives a visualization of energetically allowed and disallowed dihedral angles of amino acids, calculated based on the van der Waal radius of the side chain. 


\section{Prediction of LBL Epitopes in the Vaccine Protein}

The presence of both linear and discontinuous B-cell epitopes was predicted using ElliPro as above. It has been estimated that greater than $90 \%$ of B-cell epitopes are discontinuous; that is, their segments are distant from each other in their primary structure, but are brought close to each other upon the folding of the protein ${ }^{63,64}$.

\section{In Silico Cloning of Designed Vaccine Candidate}

The vaccine protein sequence was submitted to the JAVA Codon Adaptation Tool $(\mathrm{JCAT})^{27}$ to adapt the codon usage to $E$. coli K12. The options to avoid rho-independent transcription termination, prokaryote ribosome binding site, and restriction enzyme cleavage sites were selected. Xhol and Xbal restriction sites were added to the $\mathrm{C}$ and $\mathrm{N}$ termini of the sequence, respectively. The final nucleotide sequence was then cloned into the pET-30a (+) vector using the SnapGene 5.1.3 software.

\section{Molecular Docking of the Vaccine Construct with TLR2/TLR4}

The ability for COVCCF to generate an immune response depends on its ability to interact with immune receptors. Toll-like receptors 2 and 4 (TLR2, TLR4) are members of the TLR family, which play a role in pathogen recognition and activation of innate immunity. Therefore, the ability for COVCCF to interact with these receptors is key to the immune response. The adjuvant was selected as the region of interest, as it has been shown to be a TLR agonist ${ }^{26}$. CPORT ${ }^{25}$ was used to initially predict residues which could be 
involved in the protein-protein interaction. The results from this initial prediction were imported into the HADDOCK 2.4 server $^{24}$ for data-driven protein-protein docking. HADDOCK (High Ambiguity Driven protein-protein DOCKing) uses a collection of python scripts which make use of crystal and NMR structures for structure calculations. The best structure from the best cluster was submitted to the PRODIGY (PROtein binDIng enerGY prediction) server ${ }^{65}$ to predict the binding affinities of each of the protein-protein complexes.

\section{Immune Simulation}

The immunogenicity of COVCCF was further characterized using the C-ImmSim server ${ }^{66}$. C-ImmSim uses position-specific scoring matrix (PSSM) for immune epitope prediction and machine learning to predict immune interactions. It simulates hematopoietic stem cells in the bone marrow, T-cells in the thymus, and tertiary lymphatic organs, for their immune response. It has been determined computationally that an interval of several weeks between the prime (first) and boost (all subsequent) doses of a vaccine is required to obtain optimal antibody response ${ }^{14}$. Therefore, the simulation was set to administer three injections at timesteps 1,84 , and 168 , corresponding to time $=0,4$ weeks, and 8 weeks with a total of 1050 simulation steps. Each injection contained 1000 vaccine proteins, and all other parameters were set to their defaults. A further simulation with 12 injections setting 4 weeks apart was also carried out, which would simulate repeated exposure as typically seen in an endemic area, probing the clonal selection. The Simpson Index D, a measure of diversity, was interpreted from the plot. 
Code availability. All codes written for and used in this study are available from the corresponding author upon reasonable request.

Data availability. All predicted epitopes are available from Supplemental Data S1-S5. All other data are available from the corresponding author upon reasonable request.

\section{Author Contributions}

F.C. conceived the study. W.R.M. performed all experiments and data analysis. W.R.M. and F.C. wrote and critically revised the manuscript.

\section{Acknowledgements}

We acknowledge support from the Oak Ridge Leadership Computing Facility and Ohio Super Computing resources.

Funding: This work was supported by the National Heart, Lung, and Blood Institute of the National Institutes of Health $(\mathrm{NIH})$ under Award Number R00 HL138272 and the National Institute of Aging under Award Number R01AG066707 to F.C. This work was supported, in part, by the VeloSano Pilot Program (Cleveland Clinic Taussig Cancer Institute). 
Supplementary information is available in the online version of the paper.

Competing interests. A Provisional Patent Application for COVCCF has filled by Cleveland Clinic.

\section{REFERENCES}

1. Tortorici, M. A. \& Veesler, D. Structural insights into coronavirus entry. in Advances in Virus Research vol. 105 93-116 (Academic Press Inc., 2019).

2. Hoffmann, M. et al. The novel coronavirus 2019 (2019-nCoV) uses the SARScoronavirus receptor ACE2 and the cellular protease TMPRSS2 for entry into target cells. bioRxiv 2020.01.31.929042 (2020) doi:10.1101/2020.01.31.929042.

3. Ou, X. et al. Characterization of spike glycoprotein of SARS-CoV-2 on virus entry and its immune cross-reactivity with SARS-CoV. Nature Communications 11, 1-12 (2020).

4. Xia, S. et al. Inhibition of SARS-CoV-2 (previously 2019-nCoV) infection by a highly potent pan-coronavirus fusion inhibitor targeting its spike protein that harbors a high capacity to mediate membrane fusion. Cell Research 30, 343-355 (2020).

5. Belouzard, S., Millet, J. K., Licitra, B. N. \& Whittaker, G. R. Mechanisms of Coronavirus Cell Entry Mediated by the Viral Spike Protein. Viruses 4, 1011-1033 (2012).

6. Martin, W. R. \& Cheng, F. Repurposing of FDA-Approved Toremifene to Treat COVID-19 by Blocking the Spike Glycoprotein and NSP14 of SARS-CoV-2. (2020) doi:10.26434/CHEMRXIV.12431966.V1.

7. Amanat, F. \& Krammer, F. SARS-CoV-2 Vaccines: Status Report. Immunity vol. 52 583-589 (2020).

8. Watanabe, Y., Allen, J. D., Wrapp, D., McLellan, J. S. \& Crispin, M. Site-specific glycan analysis of the SARS-CoV-2 spike. Science eabb9983 (2020) doi:10.1126/science.abb9983.

9. Watanabe, Y. et al. Vulnerabilities in coronavirus glycan shields despite extensive glycosylation. Nature Communications 11, 2688 (2020). 
10. Jespersen, M. C., Peters, B., Nielsen, M. \& Marcatili, P. BepiPred-2.0: improving sequence-based B-cell epitope prediction using conformational epitopes. Nucleic Acids Research 45, (2017).

11. Abraham, M. J. et al. GROMACS: High performance molecular simulations through multi-level parallelism from laptops to supercomputers. SoftwareX1-2, 1925 (2015).

12. Ponomarenko, J. et al. ElliPro: A new structure-based tool for the prediction of antibody epitopes. BMC Bioinformatics 9, 514 (2008).

13. Dhanda, S. K., Vir, P. \& Raghava, G. P. S. Designing of interferon-gamma inducing MHC class-II binders. Biology Direct 8, 30 (2013).

14. Castiglione, F., Mantile, F., de Berardinis, P. \& Prisco, A. How the interval between prime and boost injection affects the immune response in a computational model of the immune system. Computational and mathematical methods in medicine 2012, 842329 (2012).

15. Doytchinova, I. A. \& Flower, D. R. VaxiJen: A server for prediction of protective antigens, tumour antigens and subunit vaccines. BMC Bioinformatics 8, 4 (2007).

16. Dimitrov, I., Bangov, I., Flower, D. R. \& Doytchinova, I. AllerTOP v.2 - A server for in silico prediction of allergens. Journal of Molecular Modeling 20, (2014).

17. Sormanni, P., Aprile, F. A. \& Vendruscolo, M. The CamSol method of rational design of protein mutants with enhanced solubility. Journal of Molecular Biology 427, 478-490 (2015).

18. Buchan, D. W. A. \& Jones, D. T. The PSIPRED Protein Analysis Workbench: 20 years on. Web Server issue Published online 47, (2019).

19. Wang, S., Li, W., Liu, S. \& Xu, J. RaptorX-Property: a web server for protein structure property prediction. Nucleic Acids Research 44, (2016).

20. Yang, J. et al. The I-TASSER suite: Protein structure and function prediction. Nature Methods vol. 12 7-8 (2014).

21. Xu, D. \& Zhang, Y. Improving the physical realism and structural accuracy of protein models by a two-step atomic-level energy minimization. Biophysical Journal 101, 2525-2534 (2011).

22. Heo, L., Park, H. \& Seok, C. GalaxyRefine: protein structure refinement driven by side-chain repacking. doi:10.1093/nar/gkt458.

23. Wiederstein, M. \& Sippl, M. J. ProSA-web: interactive web service for the recognition of errors in three-dimensional structures of proteins. Nucleic Acids Research 35, 407-410 (2007). 
24. van Zundert, G. C. P. et al. The HADDOCK2.2 Web Server: User-Friendly Integrative Modeling of Biomolecular Complexes. Journal of Molecular Biology 428, 720-725 (2016).

25. de Vries, S. J. \& Bonvin, A. M. J. J. CPORT: A Consensus Interface Predictor and Its Performance in Prediction-Driven Docking with HADDOCK. PLOS ONE 6 , e17695 (2011).

26. Khatoon, N., Pandey, R. K. \& Prajapati, V. K. Exploring Leishmania secretory proteins to design $B$ and $T$ cell multi-epitope subunit vaccine using immunoinformatics approach. Scientific Reports 7, 1-12 (2017).

27. Grote, A. et al. JCat: a novel tool to adapt codon usage of a target gene to its potential expression host. doi:10.1093/nar/gki376.

28. Gori, A., Longhi, R., Peri, C. \& Colombo, G. Peptides for immunological purposes: Design, strategies and applications. Amino Acids vol. 45 257-268 (2013).

29. Kim, Y. il et al. Infection and Rapid Transmission of SARS-CoV-2 in Ferrets. Cell Host and Microbe 27, 704-709.e2 (2020).

30. Park, S. J. et al. Antiviral efficacies of FDA-approved drugs against SARS-COV2 infection in ferrets. mBio 11, (2020).

31. Daniloski, Z., Guo, X. \& Sanjana, N. E. The D614G mutation in SARS-CoV-2 Spike increases transduction of multiple human cell types. bioRxiv 2020.06.14.151357 (2020) doi:10.1101/2020.06.14.151357.

32. Koyama, T., Weeraratne, D., Snowdon, J. L. \& Parida, L. Emergence of Drift Variants That May Affect COVID-19 Vaccine Development and Antibody Treatment. Pathogens 9, 324 (2020).

33. Lee, J. et al. CHARMM-GUI Input Generator for NAMD, GROMACS, AMBER, OpenMM, and CHARMM/OpenMM Simulations Using the CHARMM36 Additive Force Field. Journal of Chemical Theory and Computation 12, 405-413 (2016).

34. Jo, S., Kim, T., Iyer, V. G. \& Im, W. CHARMM-GUI: A web-based graphical user interface for CHARMM. Journal of Computational Chemistry 29, 1859-1865 (2008).

35. Wrapp, D. et al. Cryo-EM structure of the 2019-nCoV spike in the prefusion conformation. Science (New York, N.Y.) 367, 1260-1263 (2020).

36. Best, R. B. et al. Optimization of the Additive CHARMM All-Atom Protein Force Field Targeting Improved Sampling of the Backbone $\phi, \psi$ and Side-Chain $X_{1}$ and $X$ 2 Dihedral Angles. Journal of Chemical Theory and Computation 8, 3257-3273 (2012). 
37. Hess, B., Bekker, H., Berendsen, H. J. C. \& Fraaije, J. G. E. M. LINCS: A linear constraint solver for molecular simulations. Journal of Computational Chemistry 18, 1463-1472 (1997).

38. Braga, C. \& Travis, K. P. A configurational temperature Nosé-Hoover thermostat. The Journal of Chemical Physics 123, 134101 (2005).

39. Parrinello, M. \& Rahman, A. Polymorphic transitions in single crystals: A new molecular dynamics method. Journal of Applied Physics 52, 7182-7190 (1981).

40. Verlet, L. Computer \&quot;Experiments\&quot; on Classical Fluids. I. Thermodynamical Properties of Lennard-Jones Molecules. Physical Review 159, 98-103 (1967).

41. Darden, T., York, D. \& Pedersen, L. Particle mesh Ewald: An $N \cdot \log (N)$ method for Ewald sums in large systems. The Journal of Chemical Physics 98, 1008910092 (1993).

42. Grant, O. C., Montgomery, D., Ito, K. \& Woods, R. J. Analysis of the SARS-CoV2 spike protein glycan shield: implications for immune recognition. bioRxiv 2020.04.07.030445 (2020) doi:10.1101/2020.04.07.030445.

43. Nain, Z. et al. Proteome-wide screening for designing a multi-epitope vaccine against emerging pathogen Elizabethkingia anophelis using immunoinformatic approaches. Journal of Biomolecular Structure and Dynamics (2019) doi:10.1080/07391102.2019.1692072.

44. Larsen, M. v. et al. Large-scale validation of methods for cytotoxic T-lymphocyte epitope prediction. BMC Bioinformatics 8, (2007).

45. Calis, J. J. A. et al. Properties of MHC Class I Presented Peptides That Enhance Immunogenicity. PLoS Computational Biology 9, (2013).

46. Nielsen, M., Lundegaard, C. \& Lund, O. Prediction of MHC class II binding affinity using SMM-align, a novel stabilization matrix alignment method. BMC Bioinformatics 8, 238 (2007).

47. Wang, P. et al. Peptide binding predictions for HLA DR, DP and DQ molecules. BMC Bioinformatics 11, (2010).

48. Nielsen, M. \& Lund, O. NN-align. An artificial neural network-based alignment algorithm for MHC class II peptide binding prediction. BMC Bioinformatics 10, 296 (2009).

49. Sidney, J. et al. Quantitative peptide binding motifs for 19 human and mouse MHC class i molecules derived using positional scanning combinatorial peptide libraries. Immunome Research 4, (2008). 
50. Sturniolo, T. et al. Generation of tissue-specific and promiscuous HLA ligand databases using DNA microarrays and virtual HLA class II matrices. Nature Biotechnology 17, 555-561 (1999).

51. Andreatta, M. et al. Accurate pan-specific prediction of peptide-MHC class II binding affinity with improved binding core identification. Immunogenetics 67, 641650 (2015).

52. Wold, S., Jonsson, J., Sjörström, M., Sandberg, M. \& Rännar, S. DNA and peptide sequences and chemical processes multivariately modelled by principal component analysis and partial least-squares projections to latent structures. Analytica Chimica Acta 277, 239-253 (1993).

53. Gupta, S. et al. In Silico Approach for Predicting Toxicity of Peptides and Proteins. PLOS ONE 8, e73957 (2013).

54. Luckheeram, R. V., Zhou, R., Verma, A. D. \& Xia, B. CD4 + T Cells: Differentiation and Functions. Clinical and Developmental Immunology 2012, 12 (2012).

55. Dhanda, S. K., Gupta, S., Vir, P. \& Raghava, G. P. S. Prediction of IL4 Inducing Peptides. Clinical and Developmental Immunology 2013, 263952 (2013).

56. Nagpal, G. et al. Computer-aided designing of immunosuppressive peptides based on IL-10 inducing potential. Scientific Reports 7, (2017).

57. Gu, Y. et al. Vaccination with a paramyosin-based multi-epitope vaccine elicits significant protective immunity against Trichinella spiralis infection in mice. Frontiers in Microbiology 8, (2017).

58. Gasteiger, E. et al. Protein Identification and Analysis Tools on the ExPASy Server. http://www.expasy.org/tools/.

59. Sormanni, P., Amery, L., Ekizoglou, S., Vendruscolo, M. \& Popovic, B. Rapid and accurate in silico solubility screening of a monoclonal antibody library. Scientific Reports 7, 1-9 (2017).

60. Zheng, W. et al. LOMETS2: improved meta-threading server for fold-recognition and structure-based function annotation for distant-homology proteins. Nucleic Acids Research 47, 429-436 (2019).

61. Colovos, C. \& Yeates, T. O. Verification of protein structures: Patterns of nonbonded atomic interactions. Protein Science 2, 1511-1519 (1993).

62. Chen, V. B. et al. MolProbity: All-atom structure validation for macromolecular crystallography. Acta Crystallographica Section D: Biological Crystallography 66, 12-21 (2010).

63. Barlow, D. J., Edwards, M. S. \& Thornton, J. M. Continuous and discontinuous protein antigenic determinants. Nature 322, 747-748 (1986). 
64. van Regenmortel, M. H. V. Mapping epitope structure and activity: From onedimensional prediction to four-dimensional description of antigenic specificity. Methods: A Companion to Methods in Enzymology 9, 465-472 (1996).

65. Xue, L. C., Rodrigues, J. P., Kastritis, P. L., Bonvin, A. M. \& Vangone, A. PRODIGY: a web server for predicting the binding affinity of protein-protein complexes. Bioinformatics (Oxford, England) 32, 3676-3678 (2016).

66. Rapin, N., Lund, O., Bernaschi, M. \& Castiglione, F. Computational immunology meets bioinformatics: The use of prediction tools for molecular binding in the simulation of the immune system. PLOS ONE 5, e9862 (2010). 


\section{Supporting Information}

Title: A rational design of a multi-epitope vaccine against SARS-CoV-2 which accounts for the glycan shield of the spike glycoprotein

William R. Martin and Feixiong Cheng 2020

*To whom correspondence should be addressed:

Feixiong Cheng, $\mathrm{PhD}$

Lerner Research Institute, Cleveland Clinic

Tel: +1-216-444-7654; Fax: +1-216-636-0009

Email: chengf@ccf.org

Supporting information includes 5 Supplementary Data (excel files) and 14 Supplementary figures (pdf files). 


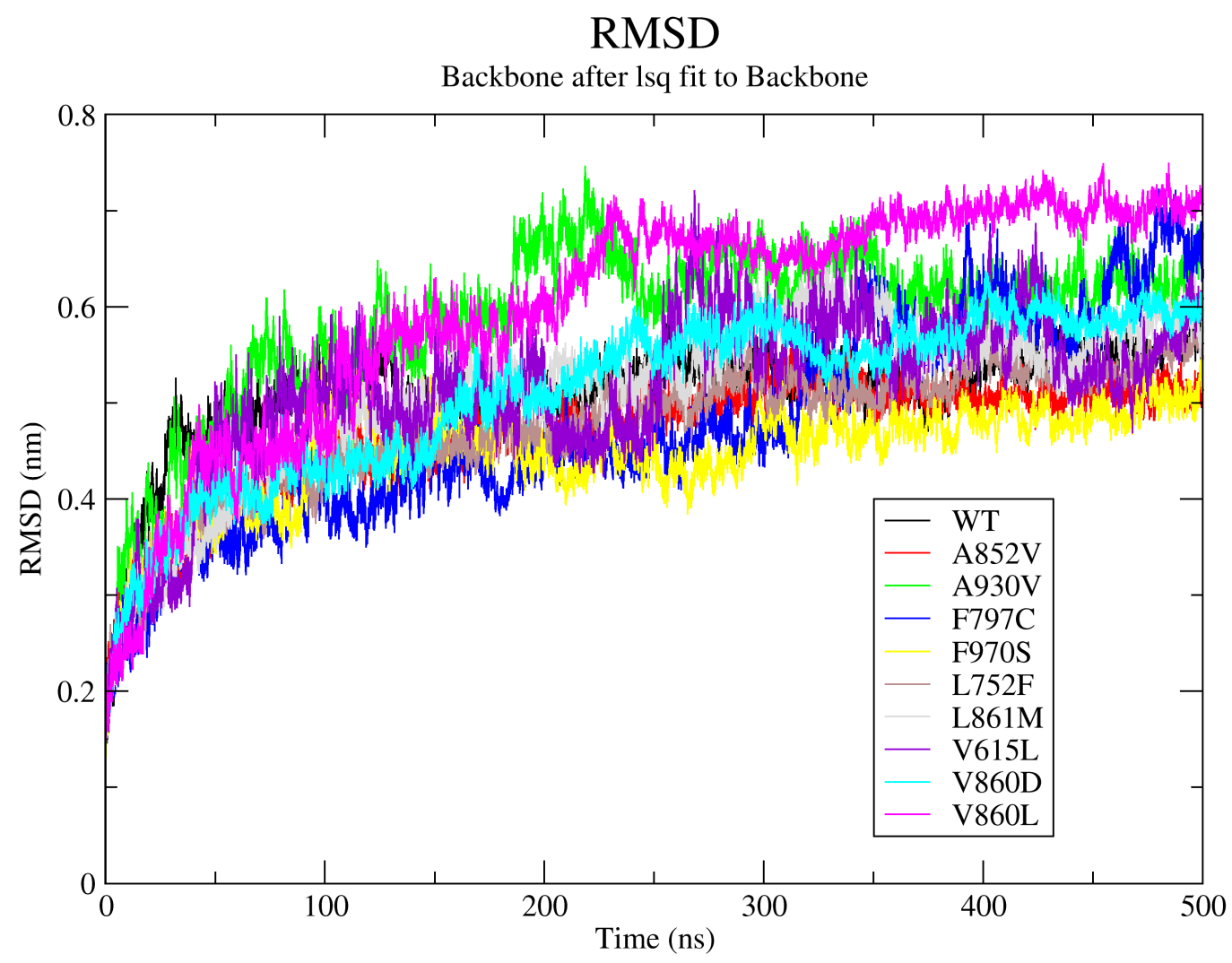

Supplementary Figure 1: Root-mean squared deviation plot over 500 nanoseconds of molecular dynamics simulation for all systems assessed for B-cell epitopes. 


\section{Area per residue over the trajectory}

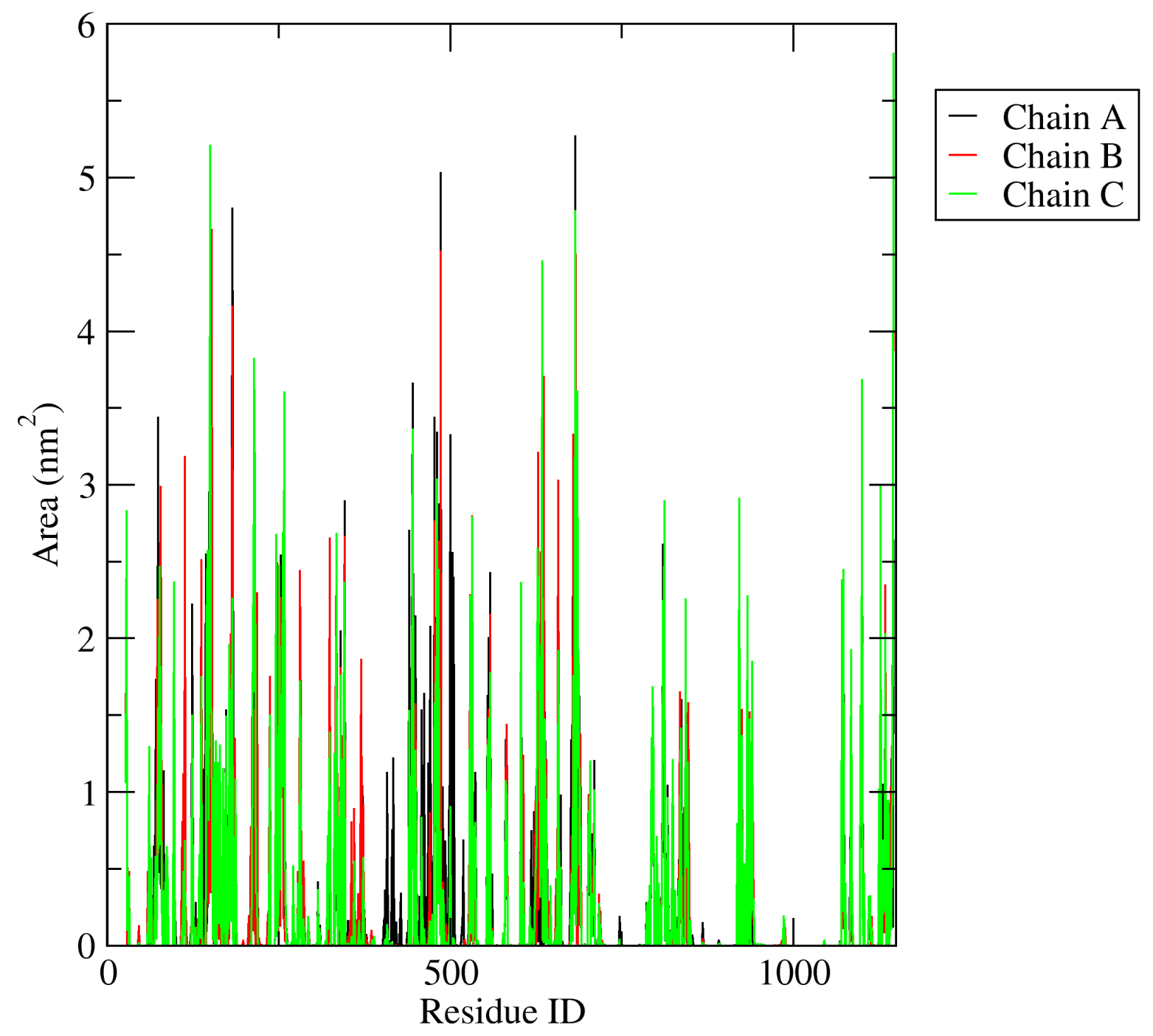

Supplementary Figure 2: Antibody-accessible surface area for the spike glycoprotein when the glycosylation is removed. 


\section{Area per residue over the trajectory}

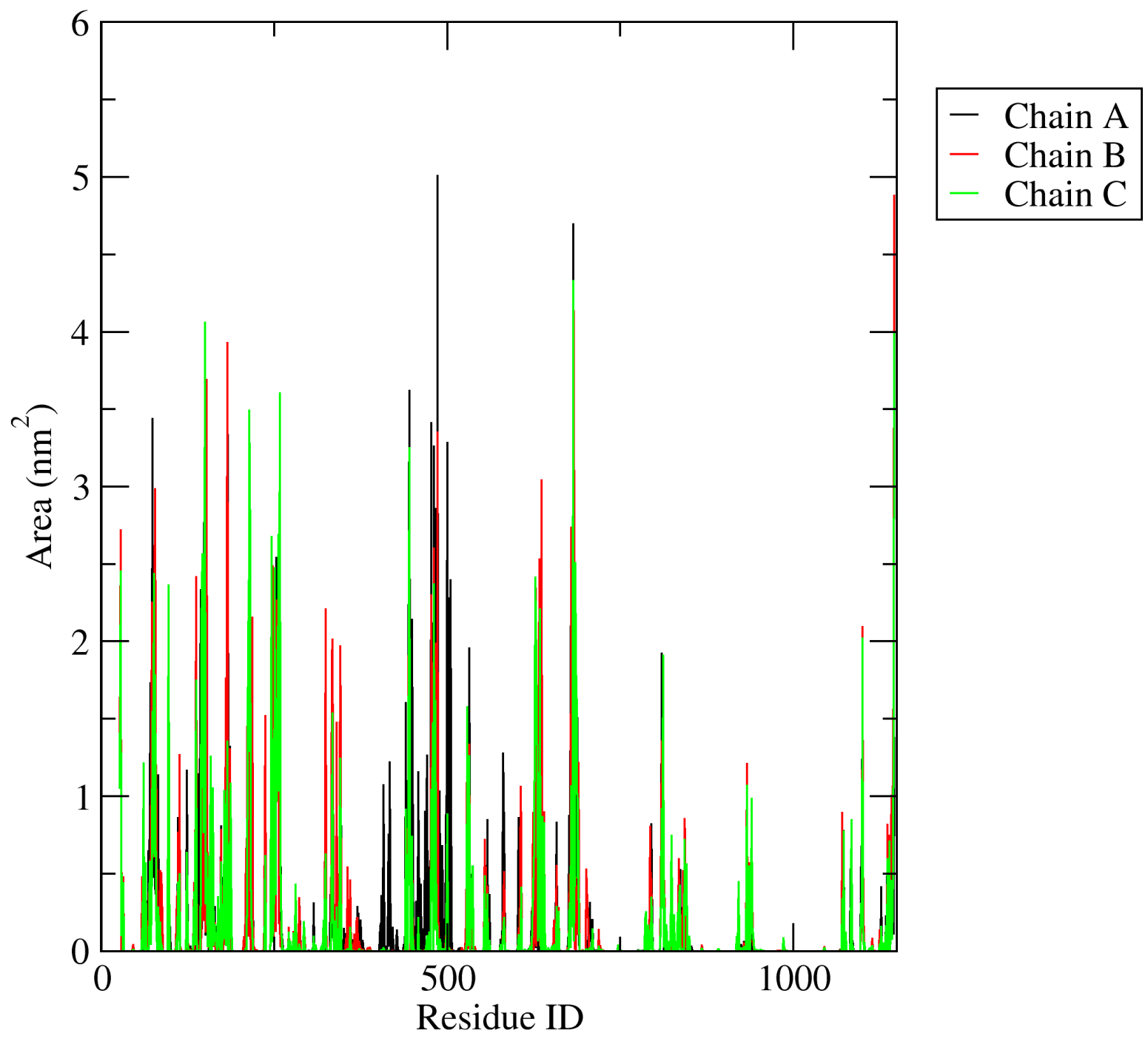

Supplementary Figure 3: Antibody-accessible surface area for the spike glycoprotein when the glycosylation is taken into account. Surface area for the glycans is not included for clarity. 


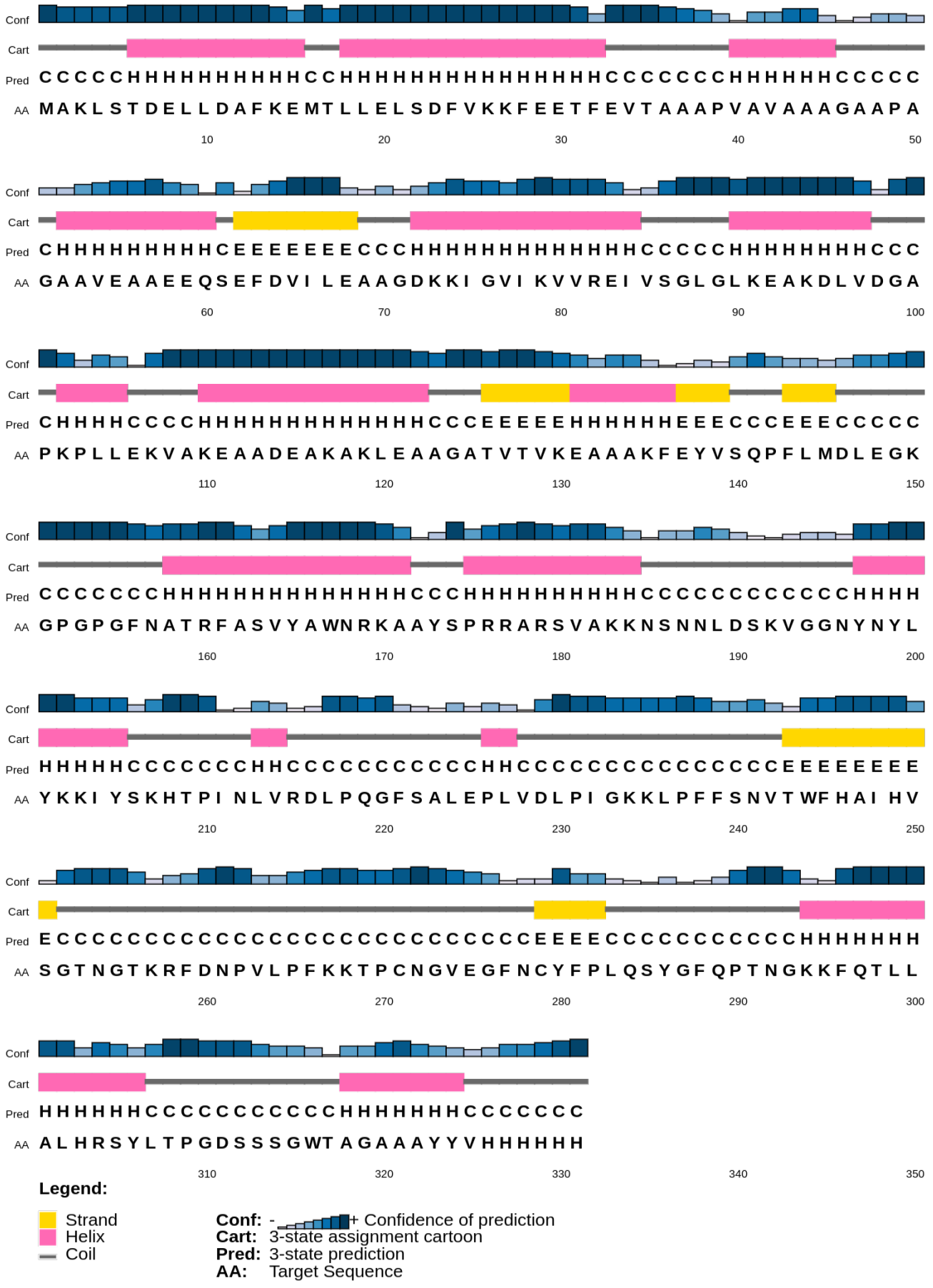

Supplementary Figure 4: Graphical representation of the predicted secondary

structure for the multi-epitope vaccine construct. PSIPRED predicts a protein with secondary structure composed of $42.6 \%$ alpha helix, $9.4 \%$ beta sheet, and $48.0 \%$ coil. 


\section{Intrinsic Disorder Profile}

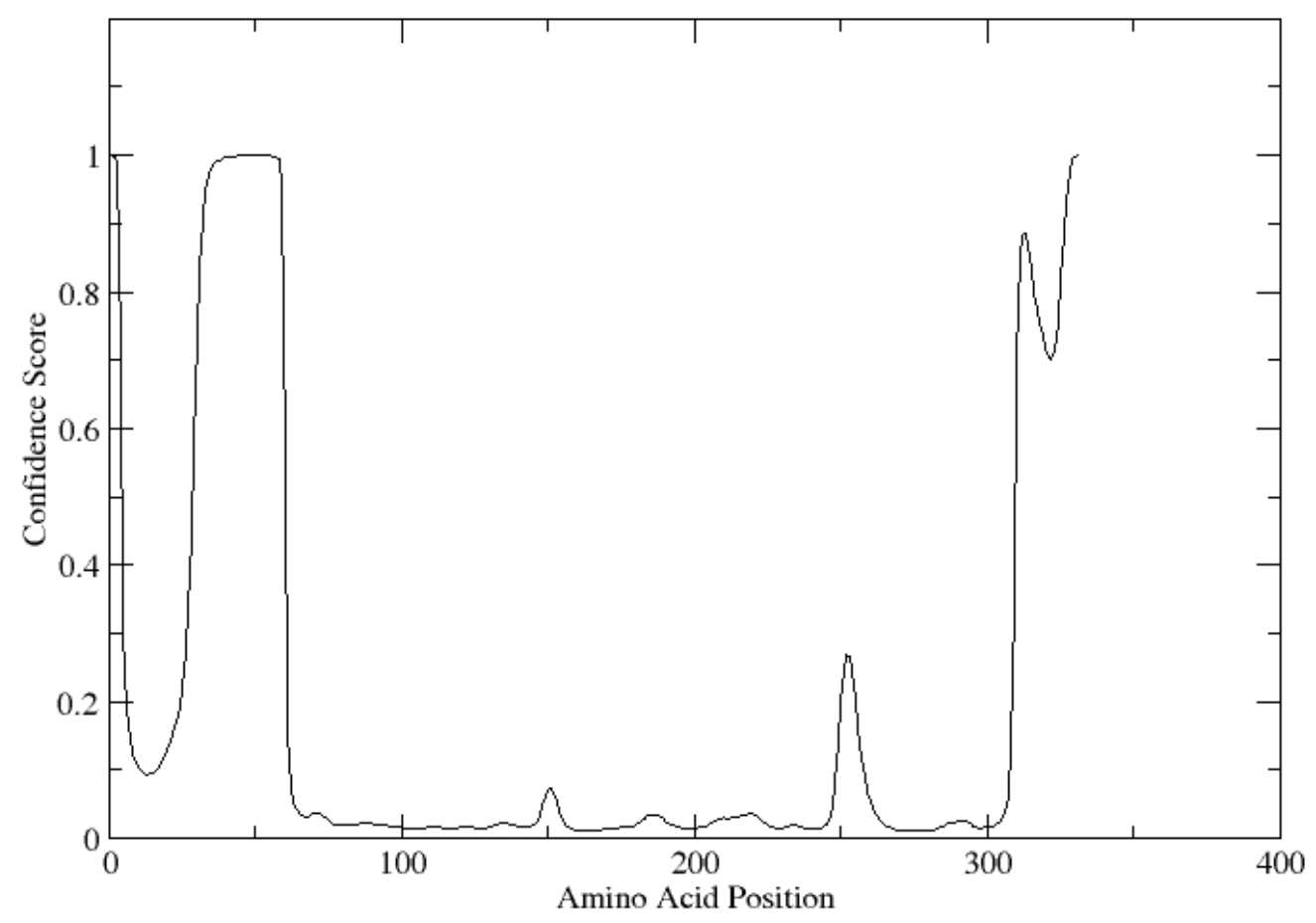

Supplementary Figure 5: Predicted disordered residue profile. 50 of the 331 residues (17\%) are predicted to be disordered by RaptorX Property. 


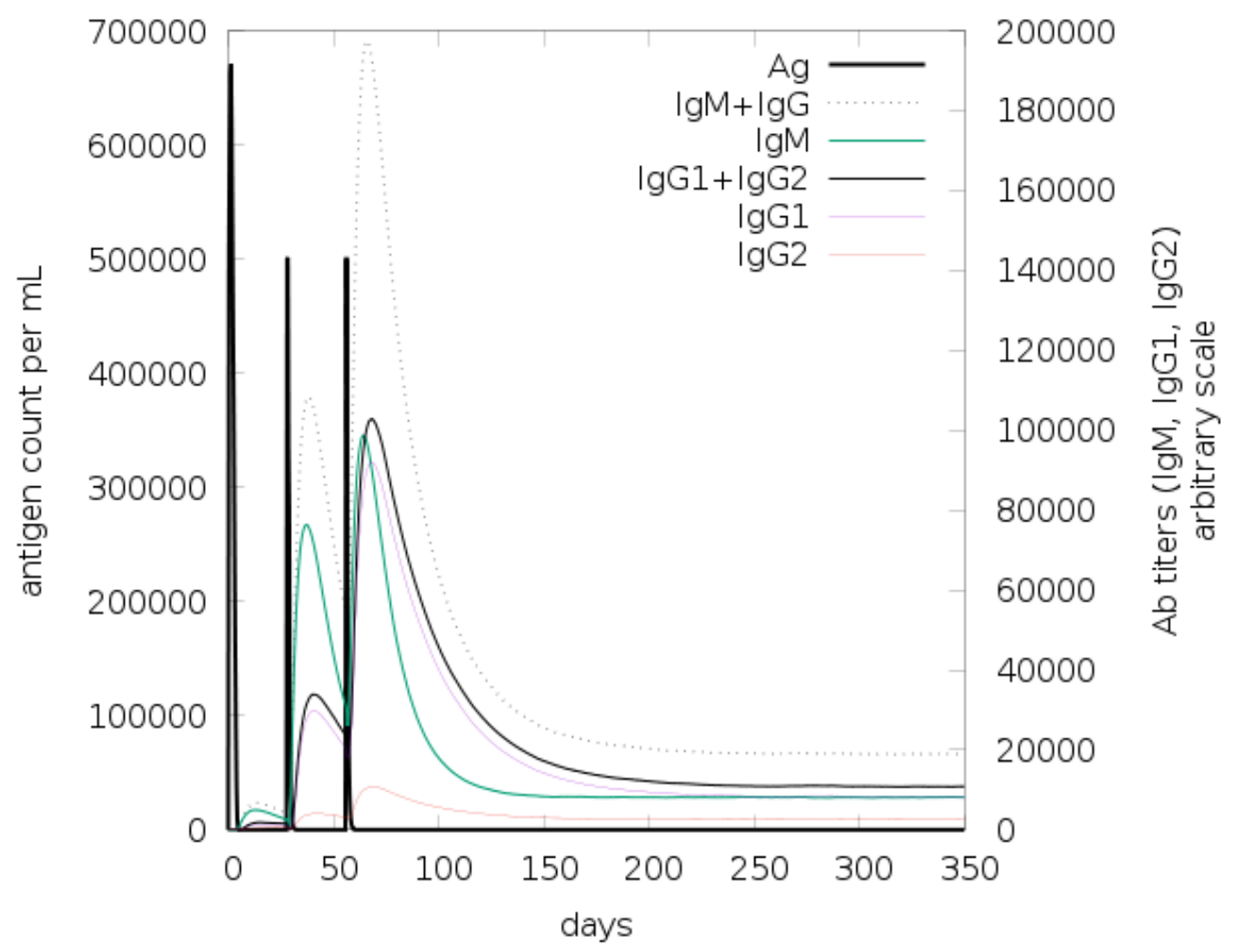

Supplementary Figure 6: Antigen and immunoglobulin levels, subdivided per isotype. 


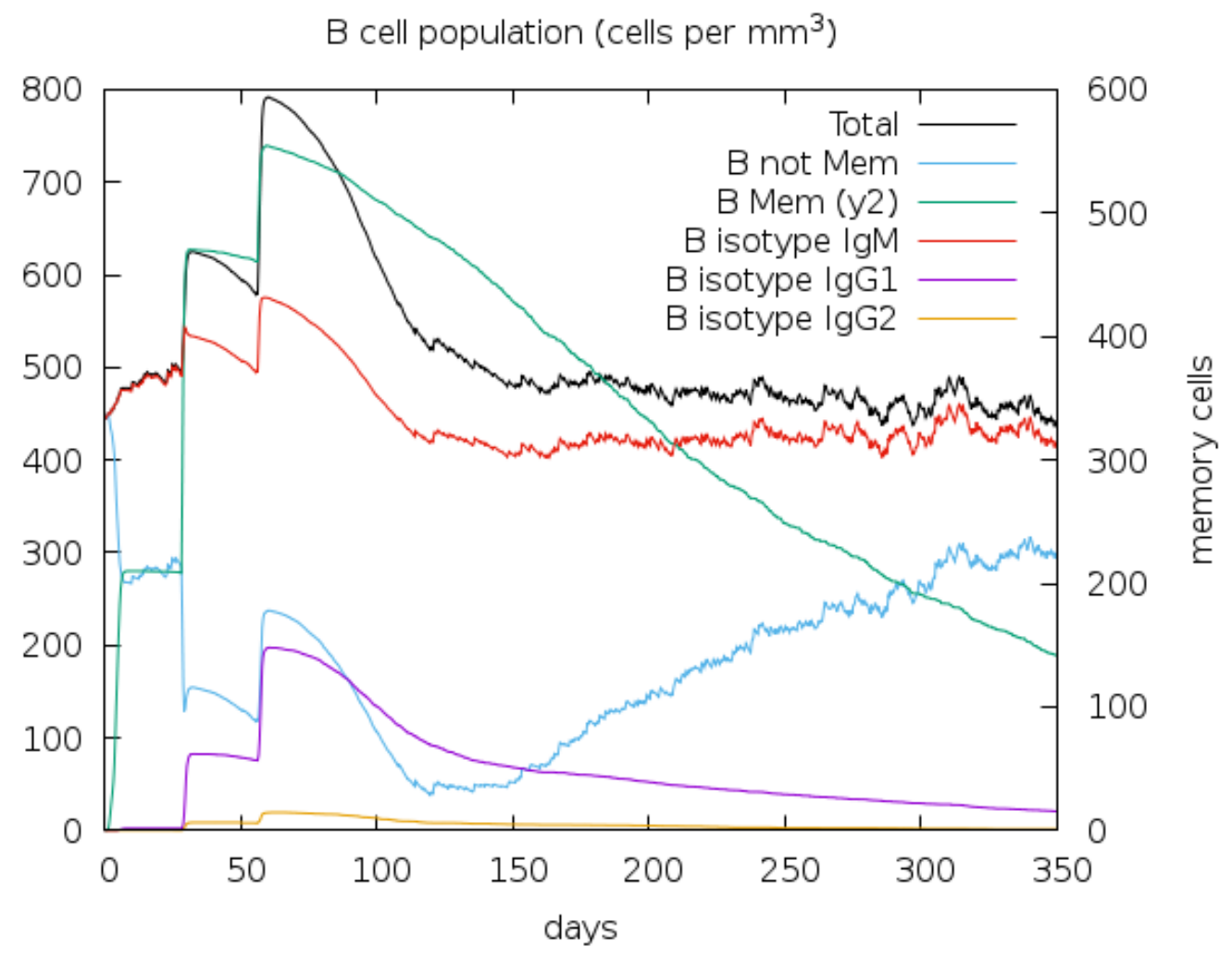

Supplementary Figure 7: B lymphocytes by total count, memory or non-memory, and isotype $(\lg M, \lg G 1, \lg G 2)$. 


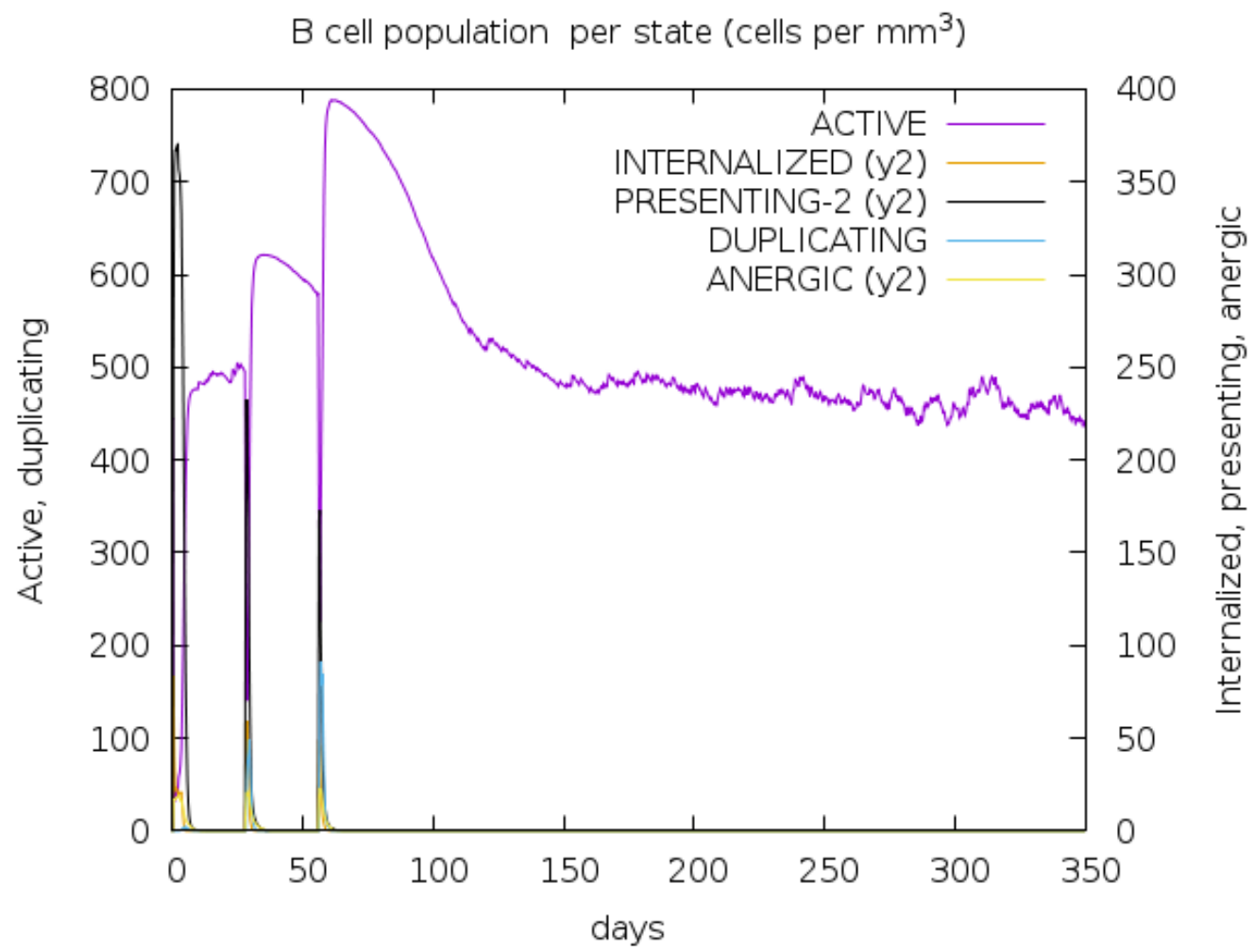

Supplementary Figure 8: B lymphocyte counts based on presentation (active, presenting on MHC class-II, internalized the antigen, duplicating, or anergic). 
TH cell population (cells per $\mathrm{mm}^{3}$ )

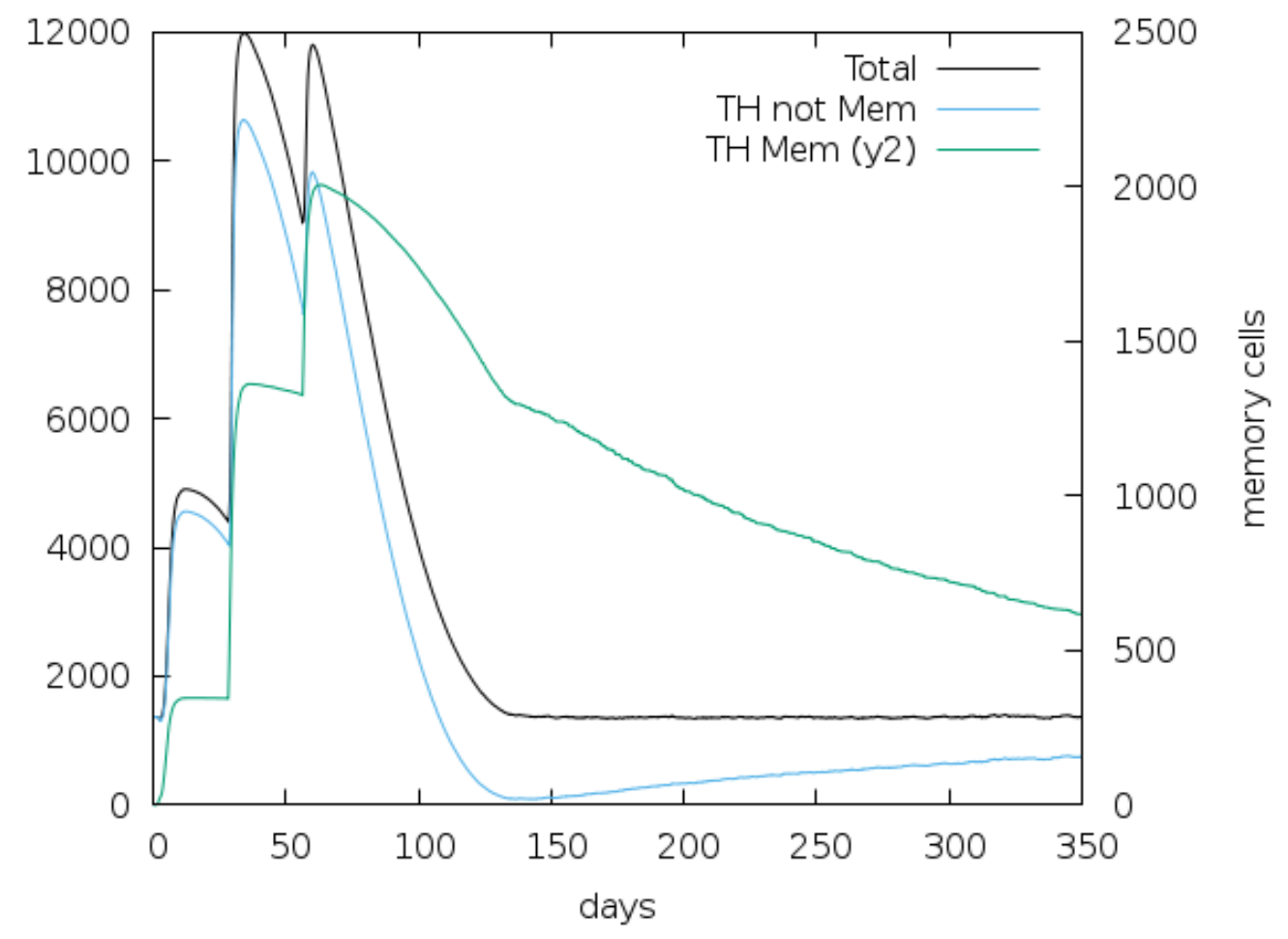

Supplementary Figure 9: Total, memory, and non-memory counts for CT4 T-helper lymphocytes. 
$\mathrm{TH}$ cell population per state (cells per $\mathrm{mm}^{3}$ )

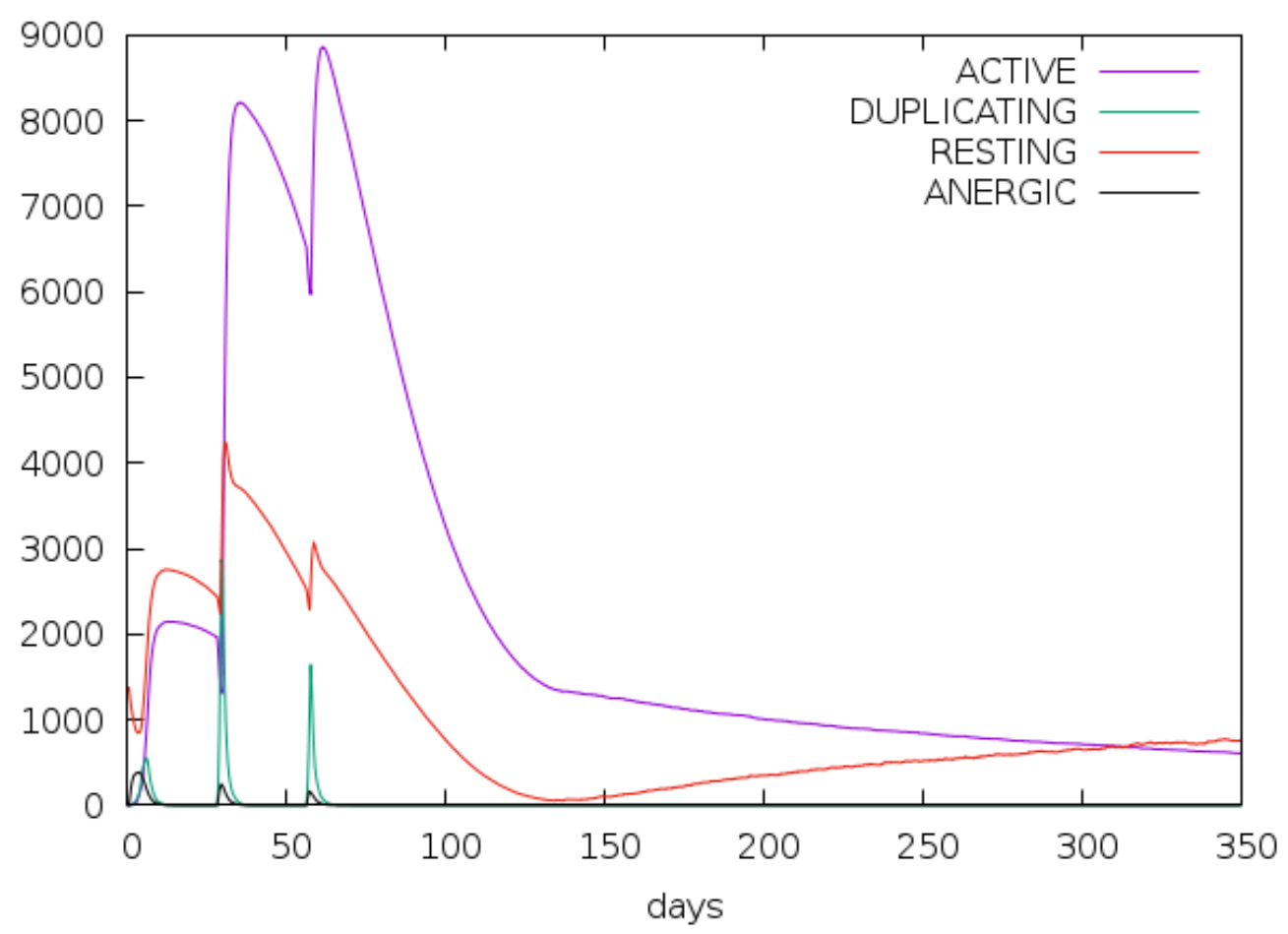

Supplementary Figure 10: Active, resting, anergic, and duplicating counts for CD4 Thelper lymphocytes. 
TC cell population (cells per $\mathrm{mm}^{3}$ )

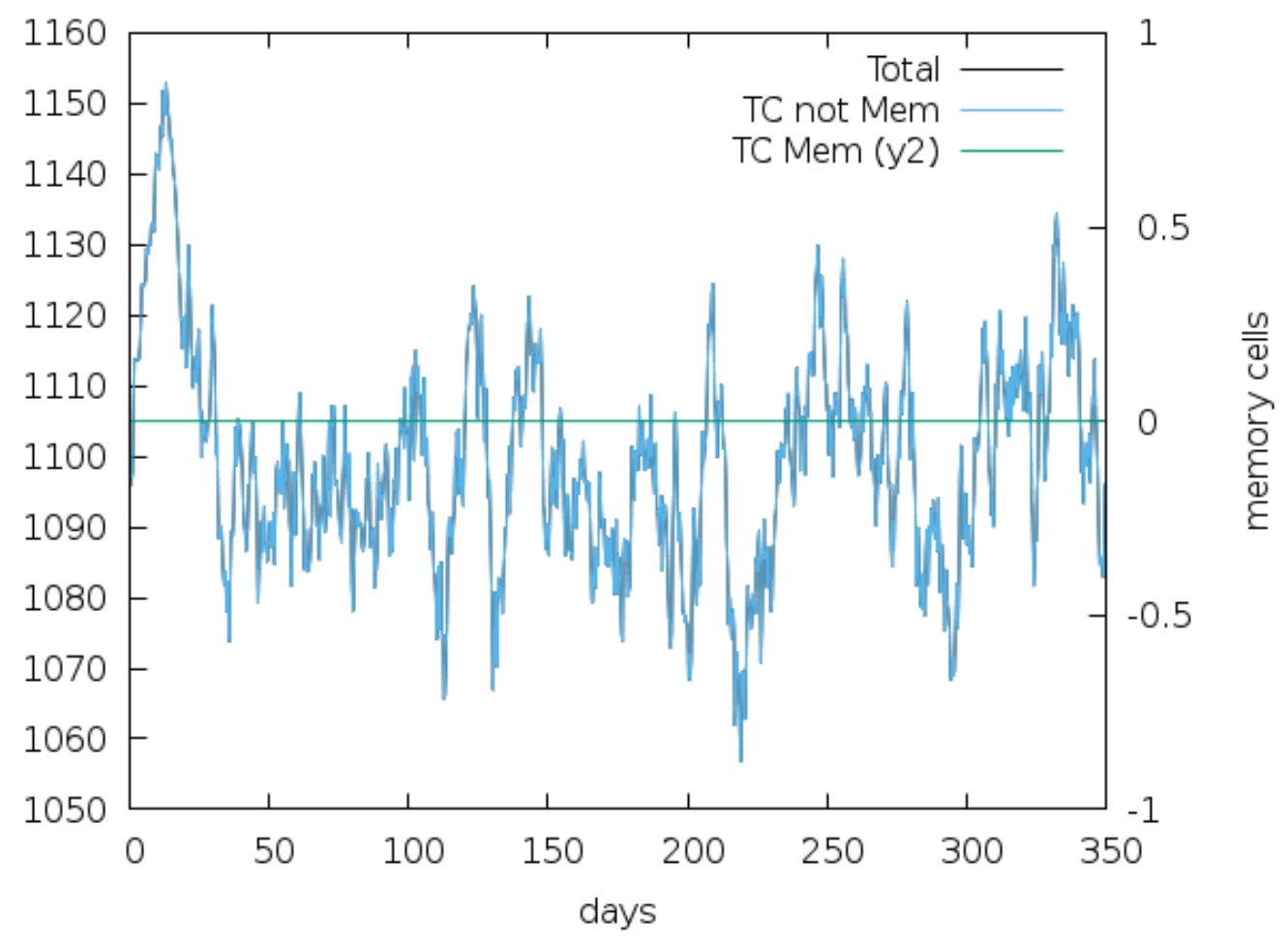

Supplementary Figure 11: Total, memory, and non-memory counts for CD8 Tcytotoxic lymphocytes. 
TC cell population per state (cells per $\mathrm{mm}^{3}$ )

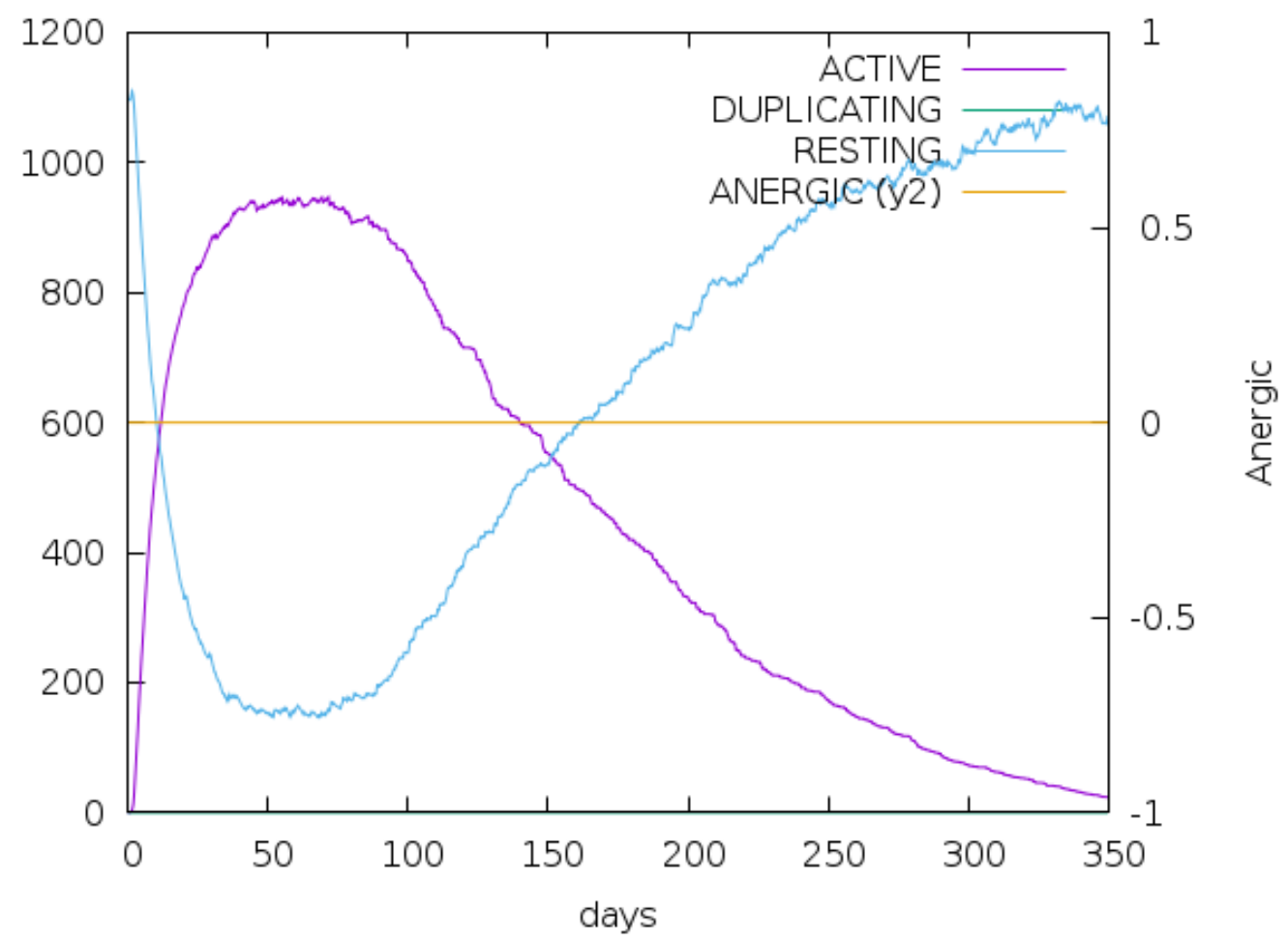

Supplementary Figure 12: Active, duplicating, resting, and anergic counts for CD8 Tcytotoxic lymphocytes. 
MA population per state (cells per $\mathrm{mm}^{3}$ )

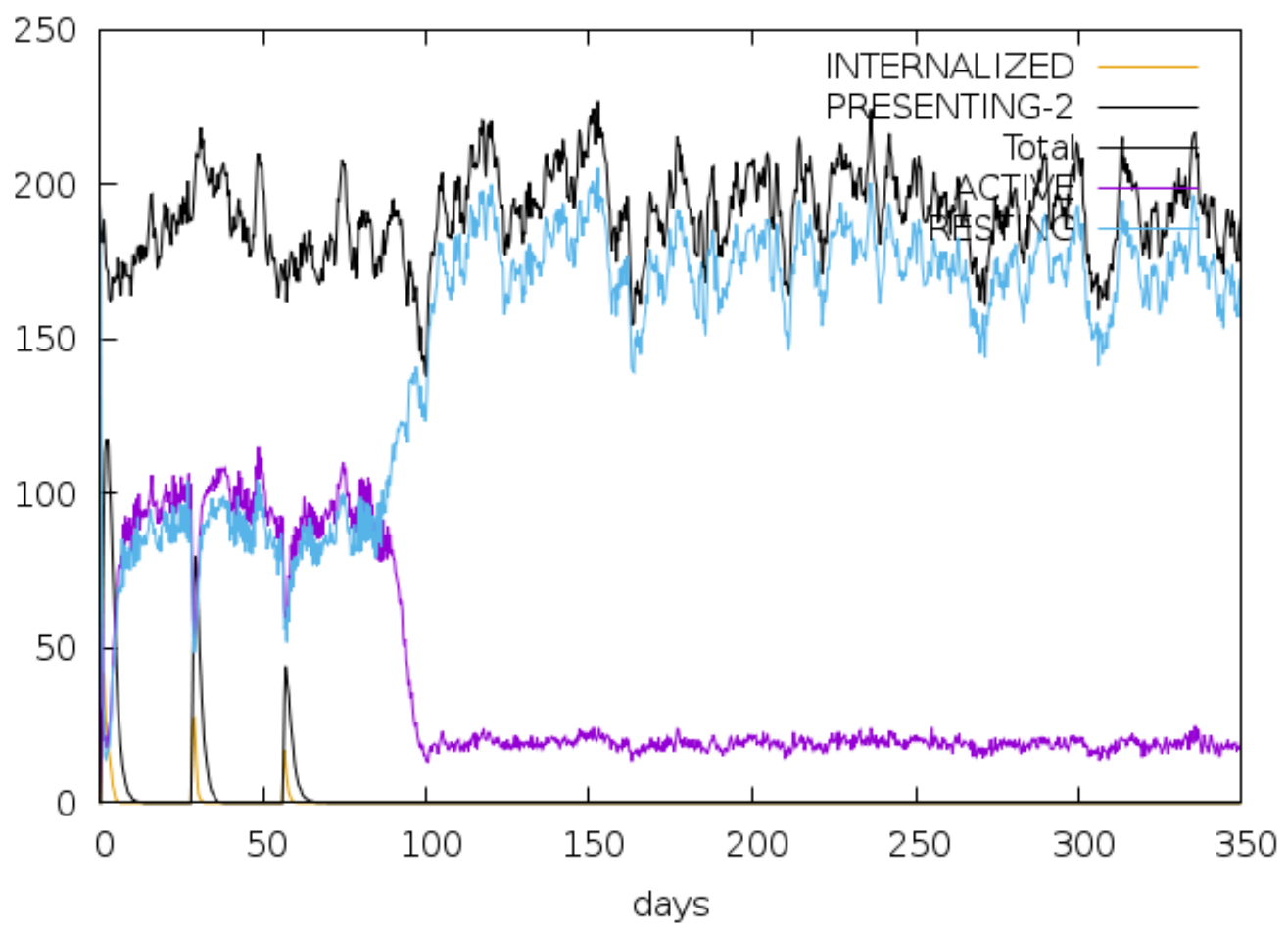

Supplementary Figure 13: Total, antigen internalized, presenting on MHC class-II, active, and resting counts for macrophages. 
DC population per state (cells per $\mathrm{mm}^{3}$ )

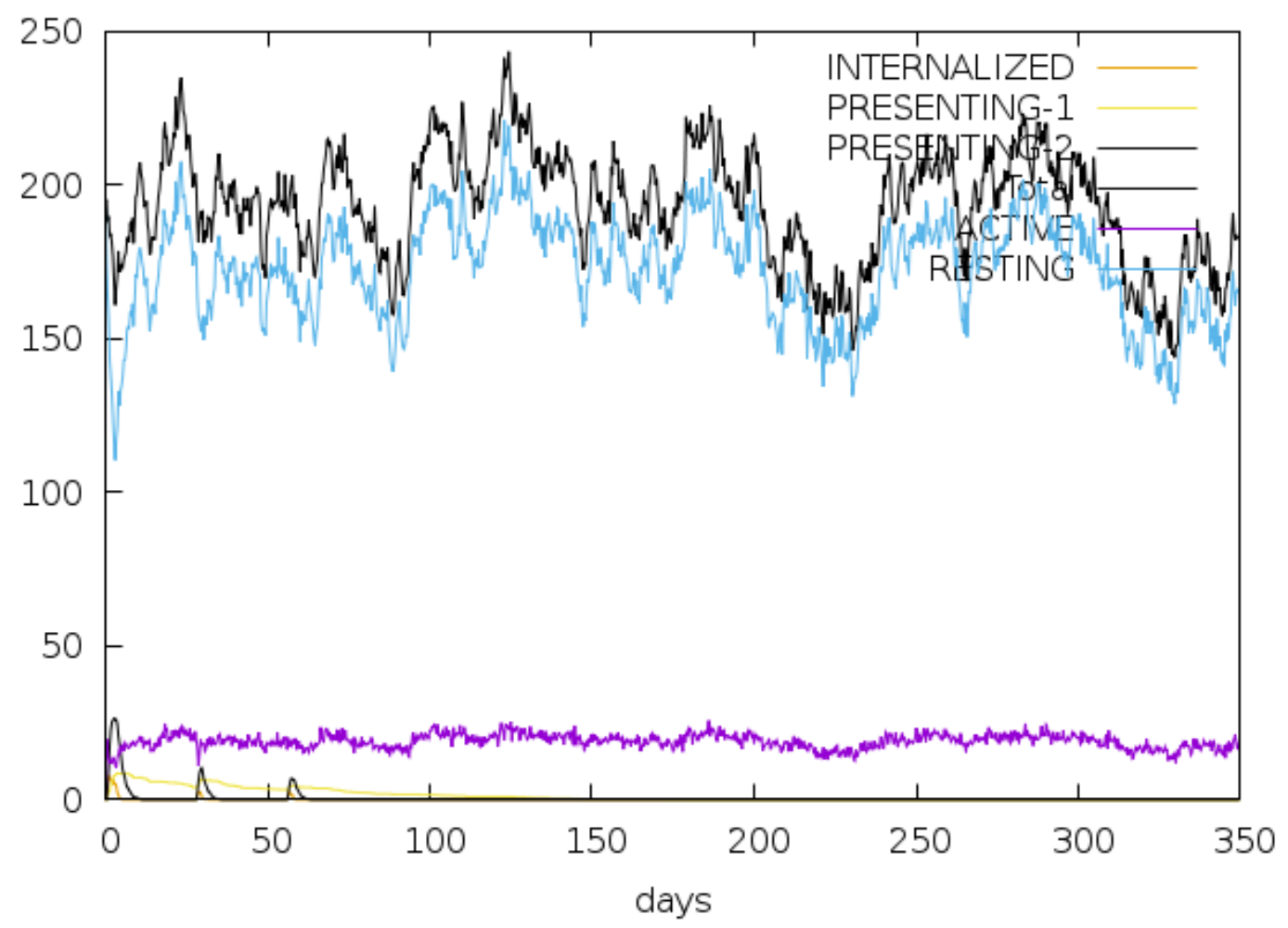

Supplementary Figure 14: Total, internalized, presenting on MHC class-I, presenting on MHC class-II, active, and resting counts for dendritic cells. 